\title{
A catalog of video records of the 2013 Chelyabinsk superbolide ${ }^{\star}$
}

\author{
J. Borovička ${ }^{1}$, L. Shrbený ${ }^{1}$, P. Kalenda ${ }^{2}$, N. Loskutov ${ }^{3}$, P. Brown $^{4}$, P. Spurný ${ }^{1}$, W. Cooke ${ }^{5}$, R. Blaauw ${ }^{6}$, \\ D. E. Moser $^{7}$, and A. Kingery ${ }^{8}$
}

\author{
1 Astronomical Institute of the Czech Academy of Sciences, 25165 Ondřejov, Czech Republic \\ e-mail: [jiri.borovicka;lukas.shrbeny]@asu.cas.cz \\ 2 Institute of Rock Structure and Mechanics of the Czech Academy of Sciences, V Holešovičkách 41, 18209 Praha 8, Czech Republic \\ 3 OOO "RC PLINOR", Shushary, Pushkinskaya 12, 196626 Saint Petersburg, Russia \\ ${ }^{4}$ Department of Physics and Astronomy, University of Western Ontario, London, Ontario, N6A 3K7, Canada \\ 5 Meteoroid Environments Office, EV44, Space Environment Team, Marshall Space Flight Center, Huntsville, Alabama 35812, USA \\ 6 All points/Jacobs ESSSA Group, NASA Marshall Space Flight Center, Huntsville, Alabama 35812, USA \\ 7 Jacobs/Jacobs ESSSA Group, NASA Marshall Space Flight Center, Huntsville, Alabama 35812, USA \\ ${ }^{8}$ ERC Incorporated/Jacobs ESSSA Group, NASA Marshall Space Flight Center, Huntsville, Alabama 35812, USA
}

Received 5 June 2015 / Accepted 11 September 2015

\section{ABSTRACT}

\begin{abstract}
The Chelyabinsk superbolide of February 15, 2013, was caused by the atmospheric entry of a $\sim 19 \mathrm{~m}$ asteroid with a kinetic energy of $500 \mathrm{kT}$ TNT just south of the city of Chelyabinsk, Russia. It was a rare event; impacts of similar energy occur on the Earth only a few times per century. Impacts of this energy near such a large urban area are expected only a few times per 10000 years. A number of video records obtained by casual eyewitnesses, dashboard cameras in cars, security, and traffic cameras were made publicly available by their authors on the Internet. These represent a rich repository for future scientific studies of this unique event. To aid researchers in the archival study of this airburst, we provide and document a catalog of 960 videos showing various aspects of the event. Among the video records are 400 distinct videos showing the bolide itself and 108 videos showing the illumination caused by the bolide. Other videos show the dust trail left in the atmosphere, the arrival of the blast wave on the ground, or the damage caused by the blast wave. As these video recordings have high scientific, historical, and archival value for future studies of this airburst, a systematic documentation and description of records is desirable. Many have already been used for scientific analyses. We give the exact locations where 715 videos were taken as well as details of the visible/audible phenomena in each video recording. An online version of the published catalog has been developed and will be regularly updated to provide a long-term database for investigators.
\end{abstract}

Key words. meteorites, meteors, meteoroids

\section{Introduction}

An extremely bright meteor, i.e. a superbolide (Ceplecha et al. 1999), appeared unexpectedly to the east of the Southern Ural mountains on the morning of February 15, 2013. The skies were mostly clear in that region and the superbolide was widely observed from the Russian regions of Kurganskaya, Tyumenskaya, Sverdlovskaya, Chelyabinskaya, Republic of Bashkortostan, Orenburskaya, Republic of Tatarstan, Samarskaya, and from the Kostanay and Aktobe regions of Kazakhstan. The blast wave produced by the superbolide caused serious damage - mostly broken windows and doors - in the city of Chelyabinsk and its surroundings and injured more than 1500 people (Popova et al. 2013; Brown et al. 2013). This unexpected and unusual event attracted worldwide attention. Scientists were eager to determine the size of the body that caused the superbolide, its velocity, trajectory, behavior in the atmosphere, the strength of the blast wave, the affected area, and other characteristics. Such a large airburst also provides ground-truth data for validation of entry models of ground damage caused by impacts, an essential component of future planetary defense efforts.

Apart from the infrasonic and seismic detections and some satellite-based observations, the main source of instrumental

\footnotetext{
* An online version of the catalog is available at http://meteor. asu.cas.cz/Chelyabinsk/
}

data proved to be videos taken by the citizens in the affected regions. These videos represent one of the largest citizen-science documentary efforts in modern times of such an unusual and transitory event. The best characterization of the trajectory, velocity, and impactor orbit were obtained using selected calibrated casual video recordings showing the bolide in flight (Borovička et al. 2013; Popova et al. 2013). Other videos documented the dust trail in the atmosphere, the arrivals of the blast waves, and the damage to buildings (Brown et al. 2013; Popova et al. 2013).

These analyses established that the superbolide was caused by an asteroid with a diameter of about $19 \mathrm{~m}$, which entered the atmosphere with the velocity of $19 \mathrm{~km} \mathrm{~s}^{-1}$. The total energy was $500 \mathrm{kT}$ TNT $\left(2 \times 10^{15} \mathrm{~J}\right)$. Chelyabinsk was the largest confirmed impact of interplanetary body since the Tunguska event in 1908, which had an estimated energy of 3-15 MT TNT (Vasilyev 1998; Boslough \& Crawford 2008).

Until recently, it was estimated from telescopic measurements of small near-Earth asteroids that on average a body with the energy of Chelyabinsk enters the atmosphere only once per century (see the curve of Harris in Brown et al. 2013). New statistics of superbolides including Chelyabinsk itself and the recent re-evaluation of asteroid discovery statistics suggest that such events are in fact more frequent and occur a few times per century (Brown et al. 2013; Harris \& D'Abramo 2015). 
Nevertheless, such events are still rare on a human lifetime scale. There was a possible 1.5 MT impact over the Indian Ocean in 1963 (Silber et al. 2009), but data for that event are only from infrasound recordings and are not as robust as the measurable ground-damage for Tunguska or the extensive records of the Chelyabinsk airburst.

Realizing that more than $90 \%$ of the Earth's surface is uninhabited or only sparsely populated and that clouds may interfere with observations, humankind can expect to wait many centuries before seeing the next event of similar energy so well documented. On the other hand, we can expect that imaging devices, both ground-based and space-borne, will be more advanced and more widespread in the future. But even if better data are obtained next time, future events will surely not be identical to Chelyabinsk. Studies of similarities and differences between airburst events, particularly those that may cause ground damage, are essential to the development of better entry models to inform authorities as a key part of mitigation efforts in the area of planetary defense.

With the new telescopic surveys, such as the ATLAS project (Tonry 2011), that are specifically designed to detect small asteroids just prior to impact it is probable in the near future that a smaller version of Chelyabinsk will be detected just prior to impact. In such a scenario, understanding the range of possible ground damage from a Chelyabinsk-like impact becomes an urgent matter of civil defense.

For all of the foregoing reasons, it is important to identify and document all available Chelyabinsk videos. Only a minority of videos documenting this airburst were likely posted on the Internet. Nevertheless, the available records represent a large amount of valuable data. It is, however, not easy to find such videos on the Internet. Their authors put them spontaneously on various video sharing servers under various names and descriptions. Although some videos became very popular and were shown in the mass media worldwide near the time of the event, many others remained largely unknown. In this paper, we present a first version of a catalog of all videos that we have been able to document and locate as of mid-2015. This catalog and its associated metadata are a result of the combined efforts of a large number of people who spent hundreds of hours searching for the videos, classifying them, removing duplicates, geolocating the cameras, and in some instances performing detailed analyses of data shown in the videos.

This version of the catalog contains 960 videos; 400 of them show the bolide, or at least part of it, in flight. In our previous work (Borovička et al. 2013) we used in situ stellar calibration, i.e. determination of the alt-azimuthal coordinate system, of 15 videos. Since that publication, we have calibrated an additional 14 videos and refined some previous calibrations. A detailed description of the calibrations will be the subject of a forthcoming paper. The purpose of this work is to provide the list of all pertinent videos and a guide to researchers using the electronic database where various ancillary data for each record is stored. We are, nevertheless, aware that our database is not complete and not all information is known for all videos (e.g. their geolocations). For this reason, we provide not only the catalog in this paper, but also maintain an online version of $i^{1}$. The online database will be updated from time to time. We encourage readers to send their additions and corrections to the second author of this paper to continue future refinement and development of the database. It is also our hope that by publishing this catalog, the owners of some videos not yet in public circulation

http://meteor.asu.cas.cz/Chelyabinsk/ may be encouraged to make their records available to the science community.

\section{The Chelyabinsk event}

Before describing the videos and the catalog, we briefly review the now well-understood and documented characteristics of the airburst event. More details can be found in Borovička et al. (2013), Brown et al. (2013), and Popova et al. (2013).

The bolide was visible for $17 \mathrm{~s}$. For the first $11 \mathrm{~s}$, it brightened almost continuously. From distances $180 \mathrm{~km}$ or less, the maximum brightness exceeded the brightness of the Sun. After the point of maximum luminosity was reached, the bolide separated into a number of distinct fragments. One fragment remained visible much longer than the others. It later landed in lake Chebarkul, $70 \mathrm{~km}$ west of Chelyabinsk. Thousands of smaller meteorites were found in a strewn field tens of kilometers long (Popova et al. 2013; Badyukov et al. 2014). A dust trail in the atmosphere formed immediately after the bolide. It gradually dispersed and remained visible for several hours. The dust trail was also clearly observed from satellites in Earth's orbit (Miller et al. 2013; Proud 2013).

A strong airblast wave reached the ground in the Chelyabinsk region about $1.5 \mathrm{~min}$ to $5 \mathrm{~min}$ after the bolide, depending on the location. The airblast was variously described by witnesses as similar to a strong sonic boom. In many places, the airblast caused windows to break, doors to blow inward, or other minor structural damage. In one individual case, the roof of a zinc plant in Chelyabinsk collapsed. The airblast wave was followed by a number of weaker secondary sonic booms. This "cannonade" lasted for several minutes in the Chelyabinsk area.

\section{Types of videos}

Most videos showing the bolide itself were obtained by dashboard cameras. The use of cameras in cars is quite common in Russia. Various types of dashboard cameras were used, some of them with high resolution (Full HD). High resolution cameras usually have a smaller field of view than low resolution ones. While most cars were moving during the bolide flight, some records were obtained from stationary cars, e.g. parked cars or cars stopped at traffic lights. Stationary records are better for calibration and determination of the trajectory of the bolide and, in particular, velocity.

Other records of the bolide came from security cameras and traffic cameras. These cameras are stationary, but are typically mounted on elevated positions and directed toward the ground, so only a small portion of the sky is typically visible. They often have low resolution and low frame rate. In some cases only secondary records taken by hand from screen monitors were published.

Many traffic/security cameras and some dashboard cameras from cars that were pointing away from the apparent location of the airburst recorded only the illumination and shadows caused by the bolide. This is also the case of cameras mounted inside buildings. Such records have proven useful for the measurement of the light curve of the bolide in its brightest phase, although care must be taken to compensate for the effects of automatic gain control (AGC) included with most cameras. The records may also contain the arrival of the blast wave. Security and traffic cameras do not have a sound track, but the arrival of the blast wave was often quite apparent by the effects on the ground: glass breaking, cameras shaking, objects moving, or even people 

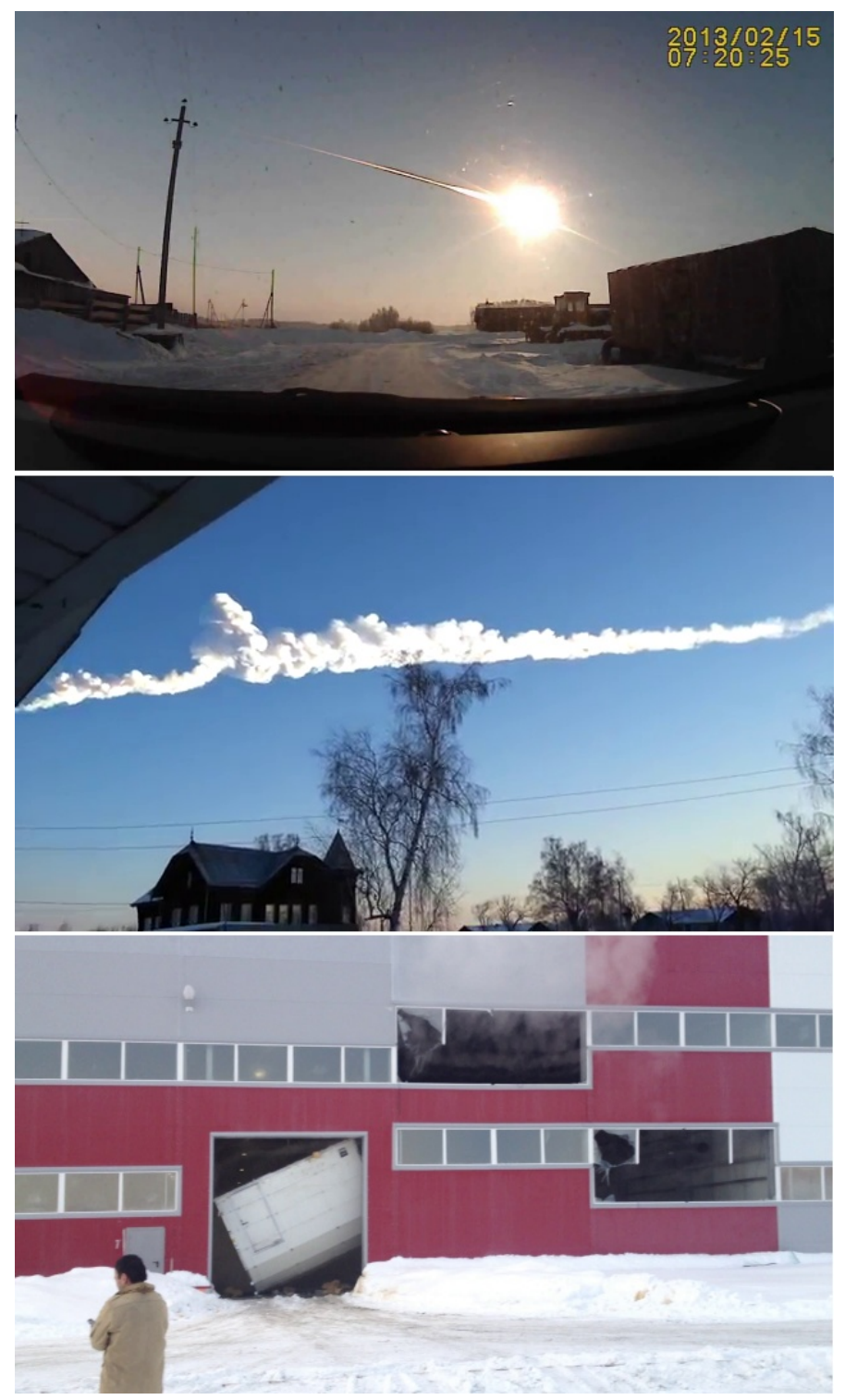

Fig. 1. Images of the bolide (top), the dust trail (middle), and damaged windows and door of a factory (bottom). The individual frames shown are from videos 137,784 , and 708, respectively. Their authors are MsANZOR95, olegfon7271, and David Částek.

falling indoors. In addition to studying the effects of the blast wave, the videos can also be useful for measuring the time interval between the bolide and the blast wave arrival and thus the origin and propagation of the wave. Of course, the time interval can be measured on many videos showing the bolide directly. Secondary booms can be studied only on records containing sound.

A large number of videos start after the bolide disappearance. These videos were typically taken by eyewitnesses to the bolide, who then decided to record the dust trail. Their videos document the dust trail, and in many cases also the arrival of the blast wave, the secondary sonic booms, and the reactions of people. Finally, some videos start only after the arrival of the blast wave. They document the damage or show the dust trail at a more evolved stage. We did not include videos showing meteorite recovery in the database.

For illustrative purposes, images from three typical videos are shown in Fig. 1.
We note that the quality of videos varies widely, not only in terms of resolution. In many cases there are missing and/or duplicated frames. Care must be therefore taken when establishing time scale on the basis of frame count. The date/time stamp embedded in the video is in most cases not correct in the absolute sense but can be used for relative timing. In a few cases the camera clock was corrected by a GPS receiver or using Internet connection.

\section{Selection of videos for the catalog}

We cataloged original videos containing a record from one camera. There are many compilations on the Internet, which combine several videos. These compilations are not listed in our catalog. The exceptions are compilations showing the records from related security cameras (e.g. from one factory) or traffic cameras (e.g. from one city). TV reports showing videos are not listed in the catalog unless they contain videos that were not found elsewhere.

The situation is somewhat complicated by the fact that many videos can be found under more than one unique internet link. Either the author uploaded multiple versions of the video (e.g. with different length or resolution) or he/she uploaded the same video on multiple video sharing servers (e.g. youtube.com and mail.ru) or other users took the original video and uploaded it again under their profiles. In these cases we list all links of the same video known to us to try to minimize issues of duplication.

In some cases the author cut the original record into two separate video sequences (e.g. one showing the bolide and one showing the blast wave) and uploaded them as two separate videos. In these cases we count them as one video and list both links.

Unfortunately, videos located on the Internet are not permanent. Videos once uploaded may later be deleted either by the author or by the provider for various reasons. In our catalog we also list links that are no longer valid. The reason is that other people may have noted these links earlier and we want to enable them to compare their list of links with ours. In some cases the video may be available under an alternative link.

Our catalog contains only videos. There are also many photos of the Chelyabinsk event on the Internet. In our experience, photos proved even more difficult to find on the Internet (there are other options for uploading photos than videos) and to geolocate than videos. Most of photos show the dust trail or damage. The photographs of the bolide in flight known to us can be found at the following links:

- http://marateaman. livejournal . com/27910.html;

- http://wwW.nashgorod.ru/users/25424/foto/ 22937/303228/;

- http://bigbangpage.com/?p=758;

- http://fineartamerica.com/featured/ meteorite-alexander-tumashov.html; and

- http://cams.seti.org/index-chelyabinsk.html.

Some photographs of the dust trail with links to original pages can be found ${ }^{2}$ in the collection of one of the team members (NL).

\section{Catalog}

The videos are listed in Tables A.1-A.4 (only available in the electronic version of the paper). Table A.1 contains 400 videos

\footnotetext{
2 At the address

http://fotki . yandex.ru/users/chelyabinskfall
} 
showing the bolide. Table A. 2 contains 108 videos showing illumination by the bolide, but not the bolide directly. Table A.3 contains 389 videos showing the dust trail in the atmosphere but neither the bolide nor the bolide light. The 63 videos in Table A.4 show either the arrival of the blast wave or damage caused by the blast wave or other consequences of the bolide. Some of them also show the dust trail in an evolved stage, i.e. a long time after the bolide, when the trail had already dispersed.

The arrangement of all four tables is the same. The following information is given in individual columns for each video in the database:

Number: the videos in Table A.1 are numbered as follows. Videos 1-15 are the calibrated videos used for the determination of the bolide trajectory by Borovička et al. (2013). Videos 16-29 are the videos calibrated later by our team that will be used for the refinement of the trajectory in a future paper. Video 30 was calibrated by Popova et al. (2013). After this video, the original database was ordered as follows: the videos with known exact locations were listed first, in the order of decreasing latitude. Then videos with known site were listed in alphabetical order according to the site. Finally, other videos were listed in alphabetical order according to the link. The numbering continued in Tables A.2-A.4, which were ordered according to the same principles except that there are no calibrated videos. Nevertheless, in the time interval between the submission and revision of this paper we were able to locate more videos and to find some new videos. In addition, ten videos were found to be duplicates and were removed from the database (the links were moved to their respective places). To avoid confusion, we are keeping the original numbering. As a consequence, some numbers are now missing and new numbers were added at the ends of Tables A.1-A.4.

Latitude and longitude: the coordinates of the position where the video was taken (eastern longitude and northern latitude in the WGS84 system). For moving cars, the coordinates at the time of bolide maximum or at another important moment in the video are given. The information on exact position was in some cases directly embedded in the video using a GPS receiver or provided by the author. Sometimes the coordinates were posted by another user. In most cases, however, we determined the actual position by comparing the landmarks visible on the video with Google Earth satellite imagery. We note, however, that a large number of videos remain with coordinates unknown and users of the database are encouraged to report updated geolocations of videos to the second author. These new locations will be updated in the online database.

Site: the name of the city or village where the video was taken. If taken in open countryside, the name of the nearest village is given, followed, if applicable, by the road number (e.g. M5). In a few cases, other geographical features are given (e.g. lake). There are also videos with unknown sites.

Camera description: for dashboard cameras, we distinguish "moving car" and "standing car". This applies to the time of the bolide or, if the bolide is not present, to another important event on the video. If the car started or stopped moving during the bolide flight, it is given as "starting car" or "stopping car", respectively. The fixed surveillance cameras are given as "security" or "traffic" according to their purpose. The keyword "time" means that the time stamp shows correct time. The note "from screen" means that secondary record from the screen monitor was published. If a multiplicity is given (e.g. $5 \times$ ), videos from multiple cameras were combined under a single link and given a single number designation in our catalog. If multiple videos under a single link were treated separately in the catalog, their order is noted after the link. The videos taken by handheld cameras (mostly by mobile phones, but sometimes also by dashboard cameras manipulated by hand) are coded "handheld".

Video description: one or more keywords are given describing the content of the video. "Whole bolide" means that (almost) the whole bolide was imaged. If only part of the bolide trajectory is visible on the video, it is specified as "beginning", "middle", or "end". If only the illumination caused by the bolide can be seen, it is given as "light". Naturally, videos showing the bolide also show the light but this is not given explicitly. For videos showing the dust trail, "trail" is given. Again, almost all videos showing the bolide also show the trail. The keyword "trail" is, however, given for videos with the bolide only in cases when more trail is visible than corresponds to the bolide part (e.g. when the dashboard camera was manually pointed to the trail or the car changed direction) or if the video is so long that it nicely shows "trail evolution". Sonic booms heard in the sound track of the video are given as "booms". If, in the absence of a sound track, the arrival of the blast wave can be seen by moving objects, it is given as "blast wave". Finally, the documentation of visible damage (e.g. broken windows) is given as "damage". We also sometimes give a note about the quality of the video (e.g. bolide overexposed).

Links: the link where the video can (or could at one time) be found. If more than one link is given for one video, the additional ones are either duplicates or related videos from the same author. We also note that more alternatives may be valid for the same link. For example these links lead to one identical video:

http: //www . youtube. com/watch?v=

REoVvY9S3Ls

http: //youtu.be/REoVvY9S3Ls

The same is true for

http://my.mail.ru/mail/dmkchel/video/

_myvideo/1.html

http://my.mail.ru/video/mail/dmkchel/

_myvideo/1.html

http://video.mail.ru/mail/dmkchel/

_myvideo/1.html

or

http: //www . odnoklassniki.ru/video/

4037216280

http://ok.ru/video/4037216280

We maintained a uniform format for all videos from the same video server in our catalog. The links that are no longer active as of July/August 2015 are given in italics in Tables A.1A. 4 .

Figure 2 illustrates the geographical distribution of videos with known locations. The distribution mostly reflects population density. The most distant bolide records came from the Samara region, more than $700 \mathrm{~km}$ from the bolide. We note that the weather was mostly cloudy to the northwest of Yekaterinburg (towards Perm) and also to the east of the easternmost observations near Tyumen. Some places to the south and east from Chelyabinsk suffered from local ground fog. Nevertheless, the skies were nicely clear in most places around the bolide trajectory. In the surroundings of Ufa, the whole bolide $(\sim 350 \mathrm{~km}$ 

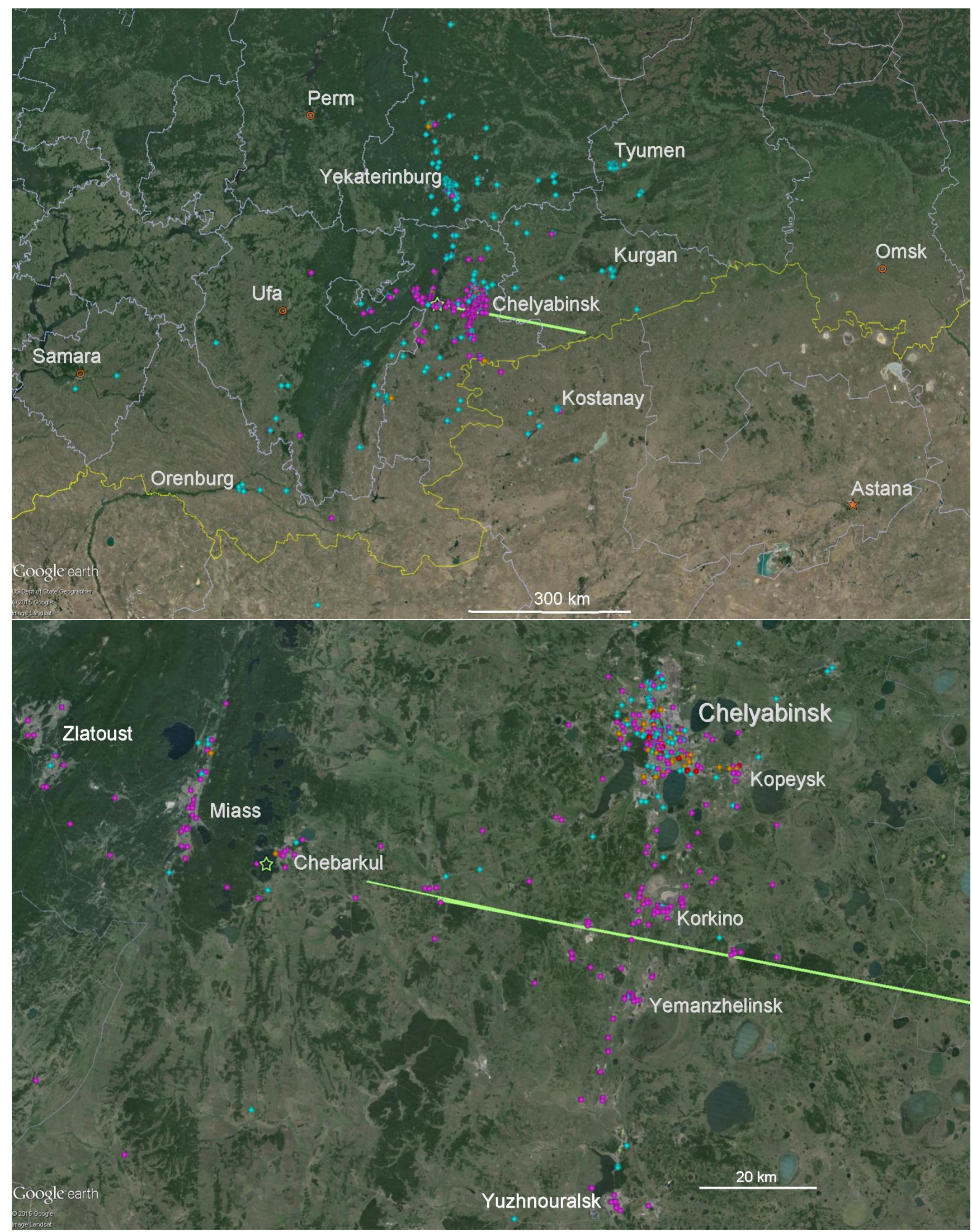

Fig. 2. Map showing the positions of 715 geolocated videos. Upper panel: overview of the whole area where the superbolide was seen; lower panel: region near the end of the bolide, including the city of Chelyabinsk. The green line is the ground projection of the bolide trajectory, including individual fragments (Borovička et al. 2013). The green star is the landing point of the largest fragment (Popova et al. 2013). The cyan dots are locations of videos showing the bolide (Table A.1). Other videos are coded as follows: orange - videos with bolide light (Table A.2), magenta - videos with trail (Table A.3), red - videos showing damage and other consequences (Table A.4). The source of the background map is Google Earth. 
away) appeared close to the rising Sun and remained mostly unnoticed.

The records of the trail are naturally more concentrated in the vicinity of the bolide, where the bolide, its acoustic effects, and the trail attracted the largest attention of the public. Nevertheless, the trail was visible at distances of more than $500 \mathrm{~km}$, e.g. from Orenburg. The sonic booms and the ground damage extended to about $100 \mathrm{~km}$ from the brightest part of the bolide, perpendicular to the trajectory (see Popova et al. 2013, for more details).

\section{Online database}

All data from Tables A.1-A.4 can be found in the online database $^{3}$. The advantages of the online version are as follows:

1. The online database will be updated from time to time when new videos or corrections to the existing items are found. Readers can report their findings by e-mail to the second author of this paper (see the e-mail address in the header of the paper). The versions of the database will be numbered and the changes made from version to version will be listed. The version published here is version number 1 .

2. A user defined table can be created by selecting the videos of a certain type (bolide, illumination, trail, wave, damage, or a combination) and columns to display. The table can be exported in text format.

3. The table can be sorted according to video number, coordinates, or site name by clicking on the table header.

4. Videos can be searched for the site name, camera type, coordinates, or a combination of these parameters.

5. The alphabetical list of all internet links in the database can be created.

6. A schematic chart showing the geographical distribution of selected videos together with the bolide trajectory (from Borovička et al. 2013) can be plotted.

The online version also contains the following additional information for the majority of the videos: resolution in pixels, number of frames per second, and the time of interest, i.e. the time, counted from the beginning of the sequence, when the bolide and/or the blast wave occurred.

\section{Summary}

We have developed a catalog of almost a thousand distinct casual video records of the Chelyabinsk superbolide of February 15, 2013, which we have found on the Internet. The Chelyabinsk event was extraordinary in the sense that a bolide of such energy (500 kT TNT) occurs only a few times per century on the whole Earth and that it was well documented. It is also a good example of the damage caused by a cosmic body. Fortunately, there were no fatalities. Video records represent the richest part of the documentation. We believe that our catalog will be useful for studying the various aspects of the event. A subsequent paper providing the calibration of selected videos and a detailed description of the bolide's trajectory and fragmentation is in preparation.

Acknowledgements. We thank L. Kotková and J. Kotek for programming the online database, H. Zichová for inspecting many videos, O. Popova for an early exchange of information on some videos, D. Ćástek for providing his video in full resolution, and authors of all videos for uploading them on the Internet. This work was supported by grant P209/11/1382 from GAČR, Praemium Academiae of the Czech Academy of Sciences, the Czech institutional project RVO:67985815, and NASA co-operative agreement NNX11AB76A.

\section{References}

Badyukov, D. D., Dudorov, A. E., \& Khaibrakhmanov, S. A. 2014, Vestnik Chelyab. Gosudar. Univ. 1/2014, Fizika, Vyp. 19, 40 (in Russian)

Borovička, J., Spurný, P., Brown, P., et al. 2013, Nature, 503, 235

Boslough, M. B. E., \& Crawford, D. A. 2008, Int. J. Impact Eng. 35, 1441

Brown, P. G., Assink, J. D., Astiz, L., et al. 2013, Nature, 503, 238

Ceplecha, Z., Spalding, R. E., Jacobs, C. F., et al. 1999, In Meteoroids 1998, eds. W. J. Baggaley, \& V. Porubčan, Astron. Inst. Slovak Acad. Sci., Bratislava, 37

Harris, A. W., \& D’Abramo, G. 2015, Icarus, 257, 302

Miller, S. D., Straka III, W. C., Scott Bachmeier, A., et al. 2013, Proc. Nat. Acad. Sci., 110, 18092

Popova, O. P., Jenniskens, P., Emel'yanenko, V., et al. 2013, Science, 342, 1069

Proud, S. R. 2013, Geophys. Res. Lett., 40, 3351

Silber, E. A., ReVelle, D. O., Brown, P. G., \& Edwards, W. N. 2009, J. Geophys. Res., 114, E08006

Tonry, J. L. 2011, PASP, 123, 58

Vasilyev, N. V. 1998, Planet. Space Sci., 46, 129

\footnotetext{
3 At the address

http://meteor . asu. cas.cz/Chelyabinsk/
}

A90, page 6 of 35 


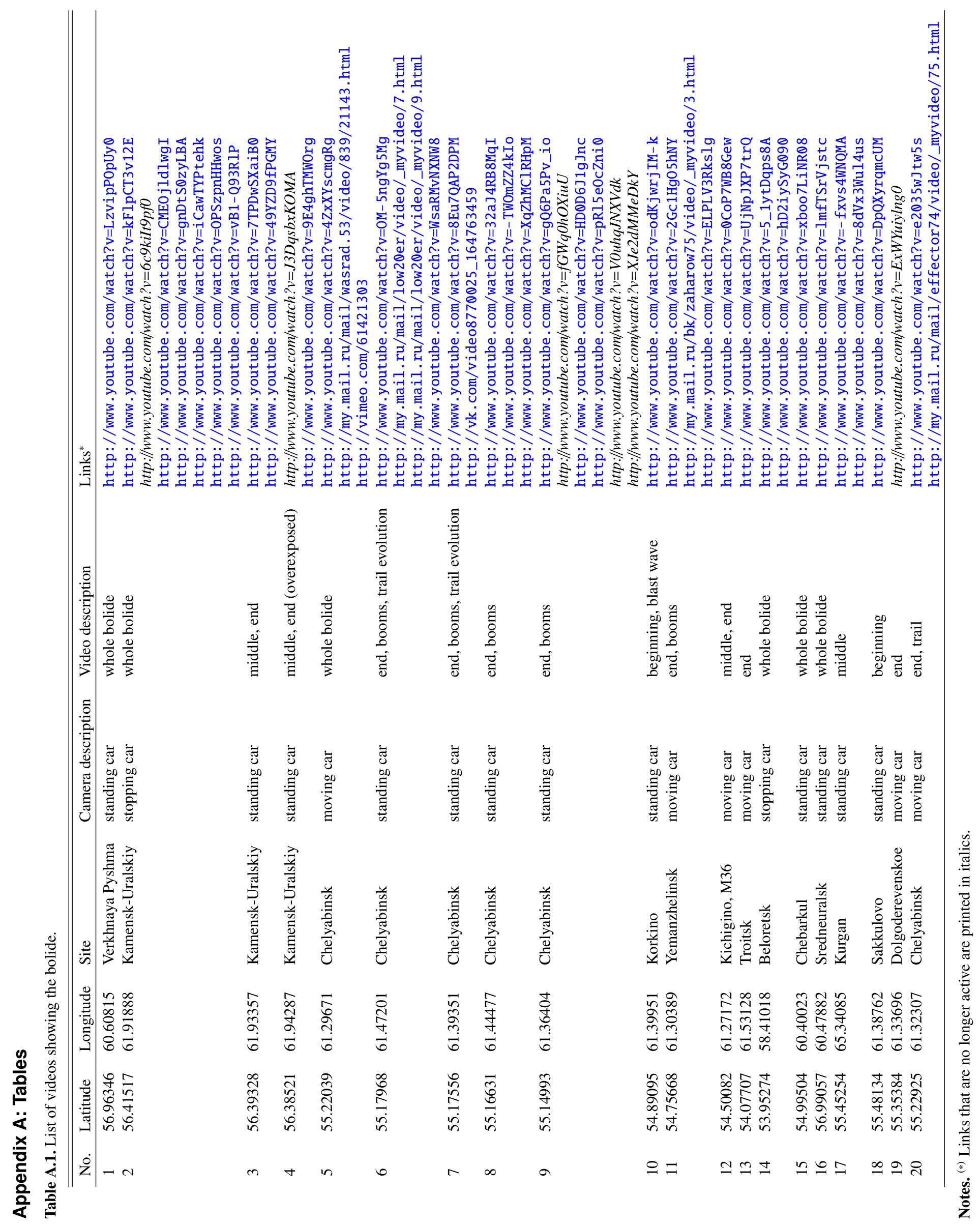




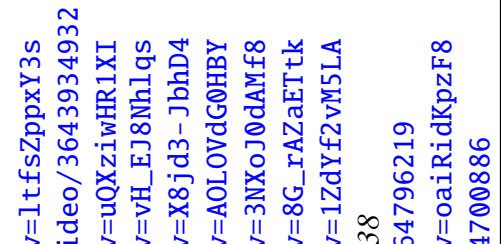

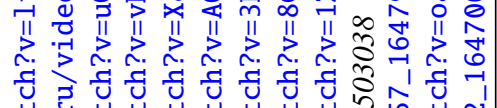

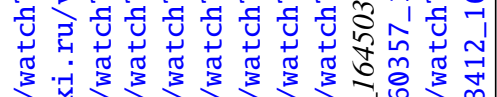
言苟言言言言言言言

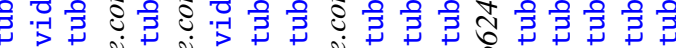

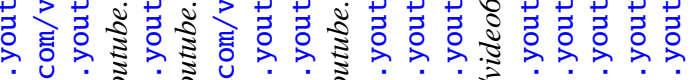
ن.

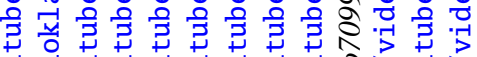

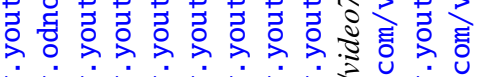

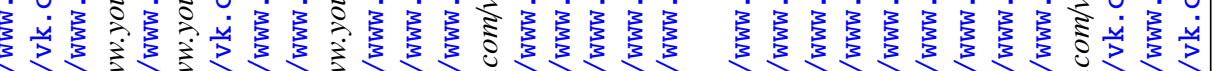

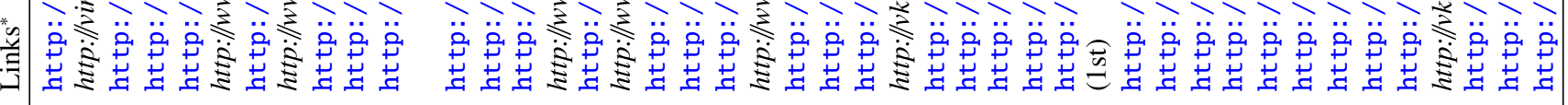

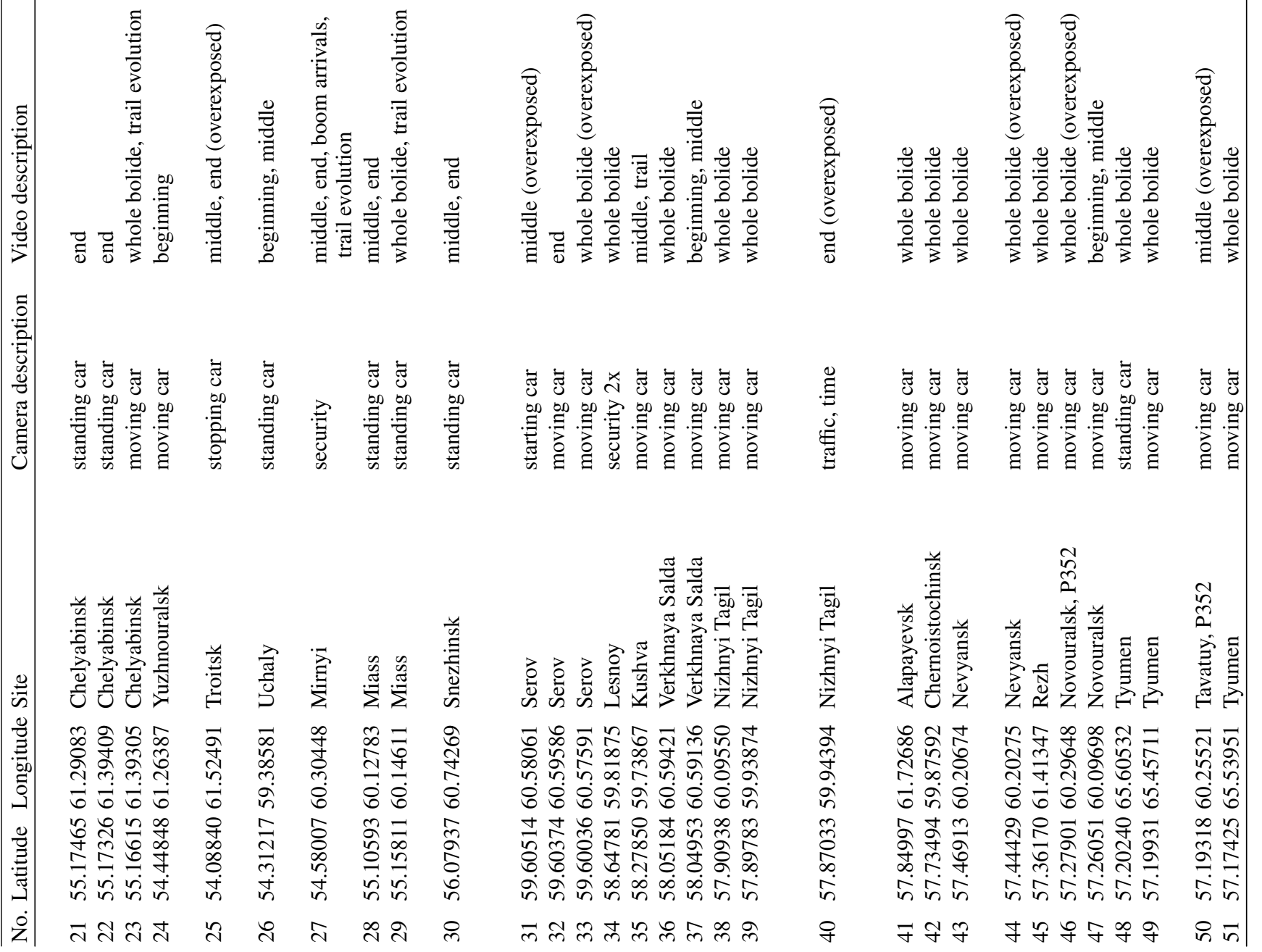


ชิ<smiles>C1CC2CC(C1)C2</smiles>

है के

竞:

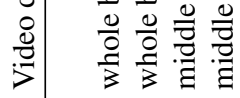

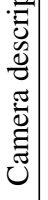

ซี ซี ซี ซี 이에 要究

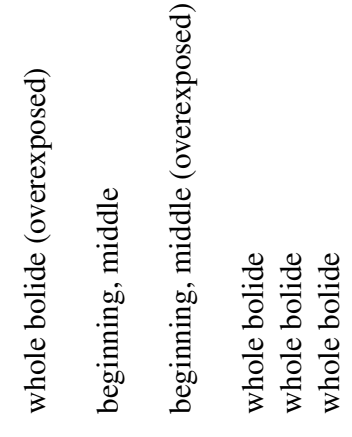

ิㅗㅇ

\section{家:}

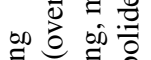

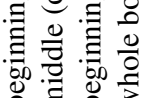

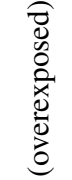

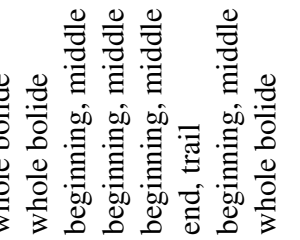

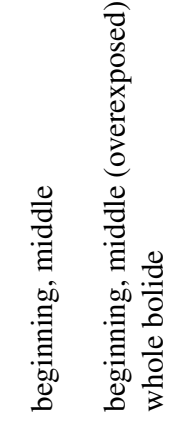

ฮี ซี

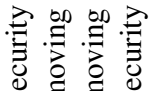

กิ

हี ซี हี 交交交架

芯胥节

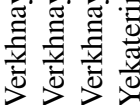

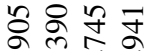

ते ते ते ชं 808 $\infty \circ 8 \infty$ 은

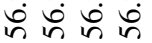
ハংঃচ 


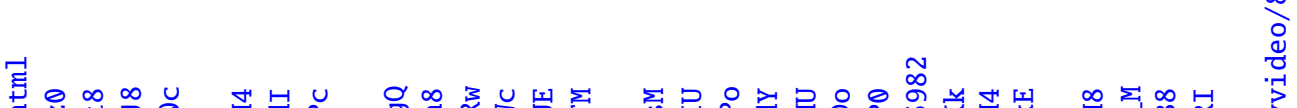

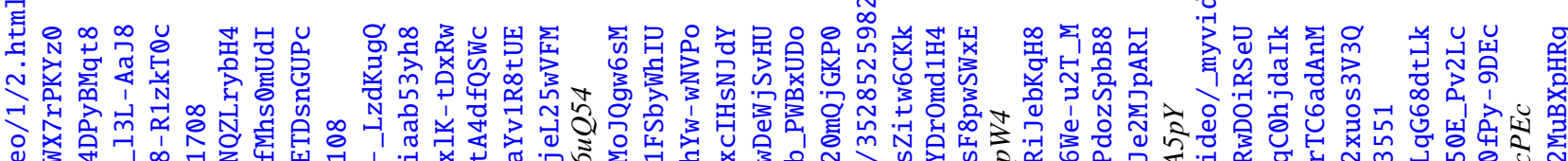

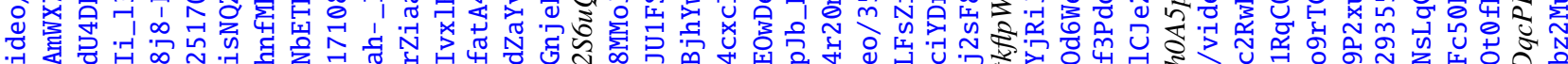

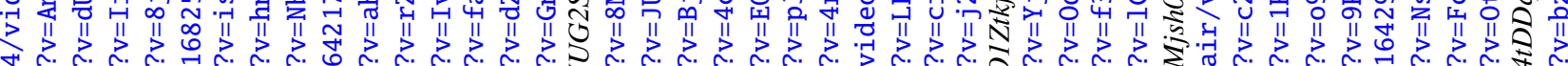

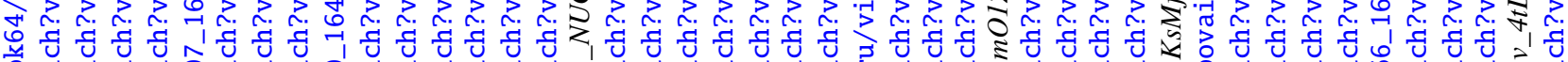

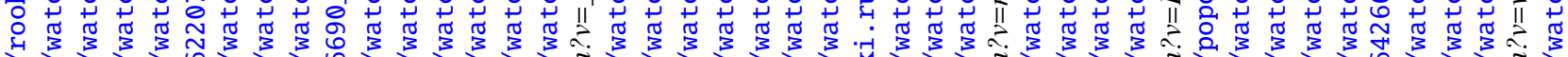

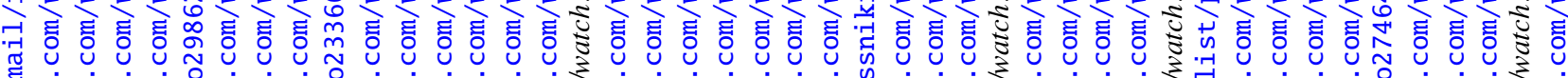

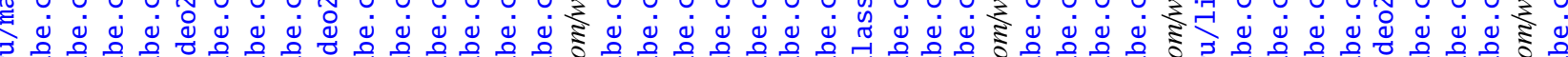

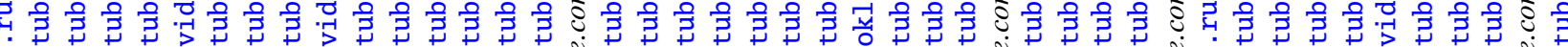

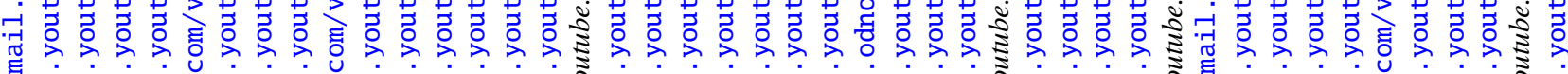

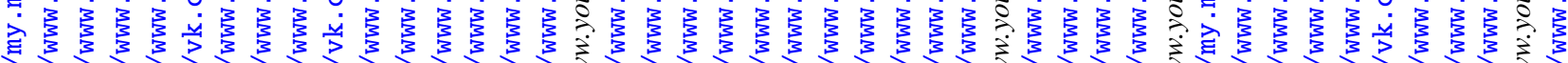
荐

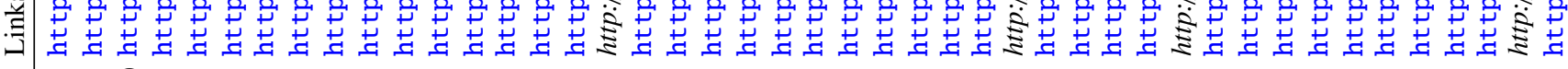

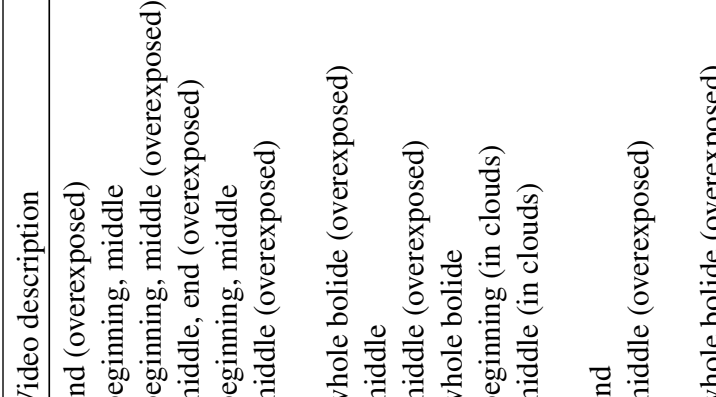

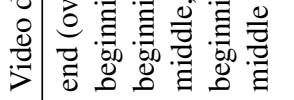

:

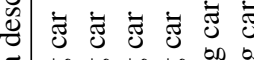

恶

$\tilde{\kappa}$

然

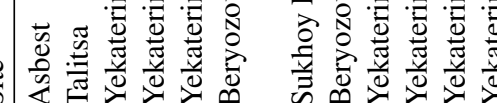

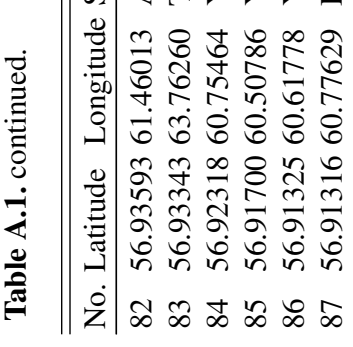

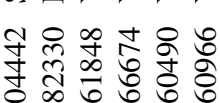
1 010 ํํㅇㅇㅇㅇㅇ

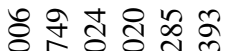
응요

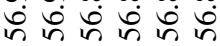

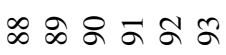

言

焉焉焉焉焉

ชิ ฮิ

ถั $\frac{\pi}{6}$

8̊

ก $ง$ ก

ले तै

棺

ปั ฉ

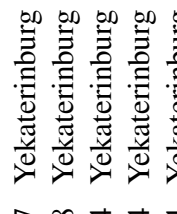

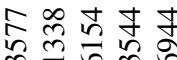

กิ $\frac{\pi}{6}$ กิ

80808

先缹尔

लत तิ तु क

ம்

ํํㅇㅇㅡ $\frac{0}{\tilde{z}}$

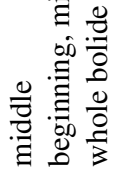

छี

焉焉

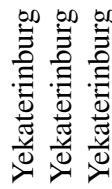

는

๑ें

휴 $\infty$

它

으 으

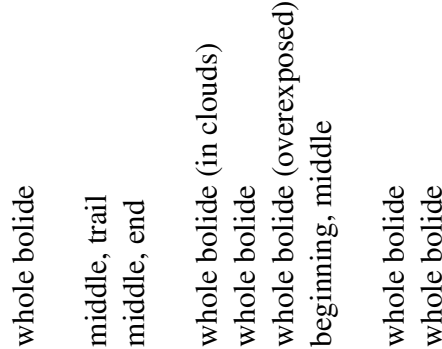

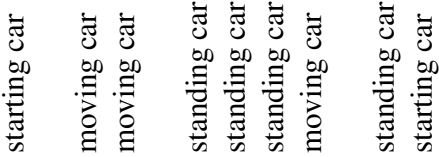

劳

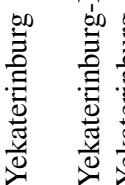

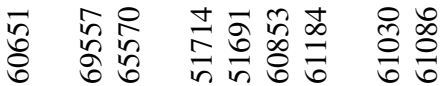

:

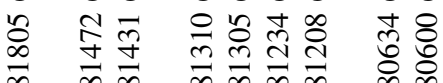

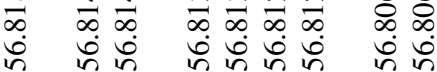

¿ 
J. Borovička et al.: A catalog of video records of the 2013 Chelyabinsk superbolide

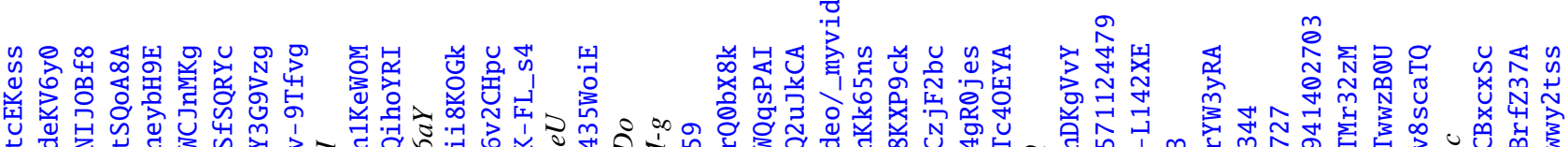

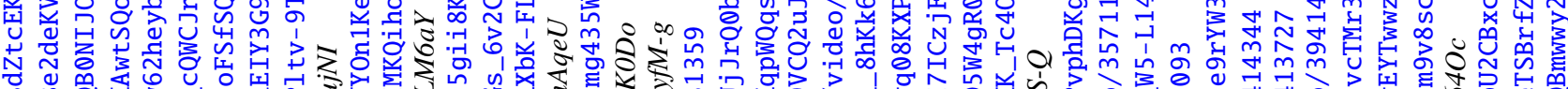

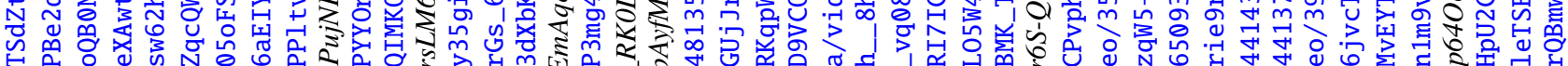

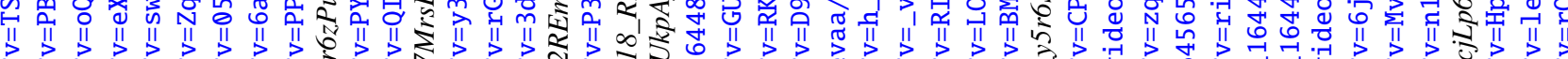

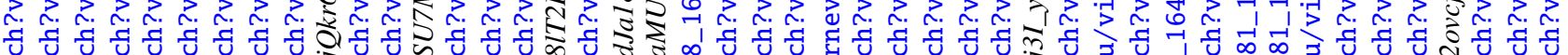

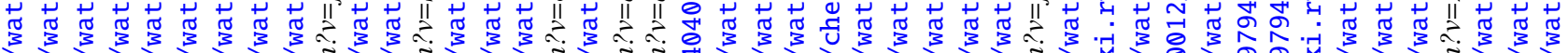

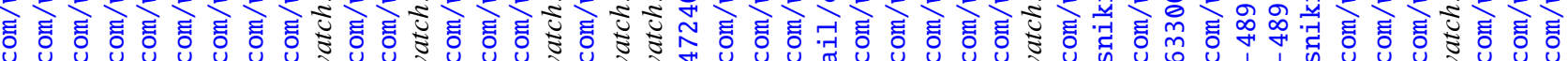

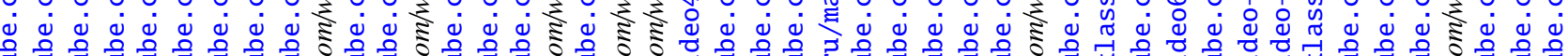

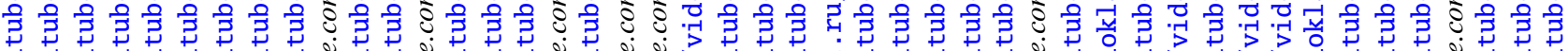

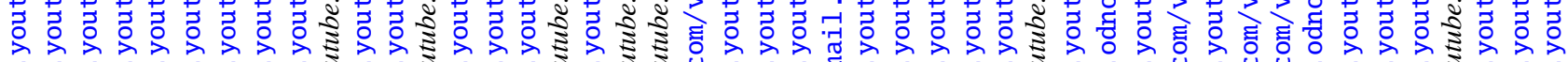

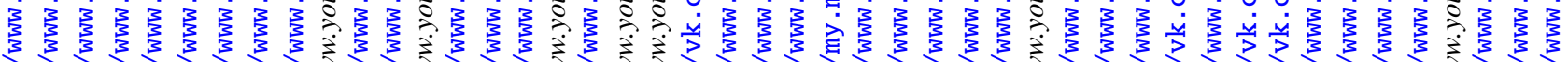

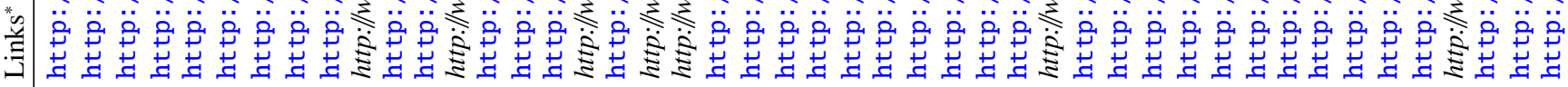

\begin{tabular}{|c|c|c|c|c|c|c|c|c|c|}
\hline 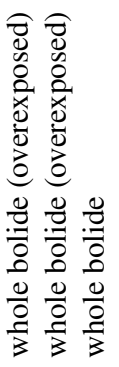 & 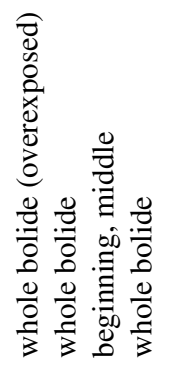 & $\begin{array}{l}\frac{0}{8} \\
\frac{0}{8} \\
\frac{0}{0} \\
\frac{\pi}{3}\end{array}$ & $\begin{array}{l}\frac{8}{0} \\
: 8 \\
\frac{0}{0} \\
\frac{0}{3} \\
\frac{\pi}{3}\end{array}$ & 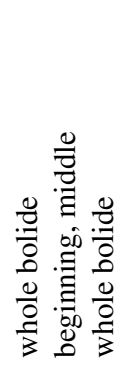 & $\begin{array}{l}\vec{\Xi} \\
\overline{0} \\
\ddot{\Xi} \\
. \bar{g}\end{array}$ & 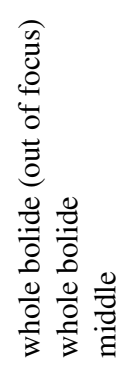 & 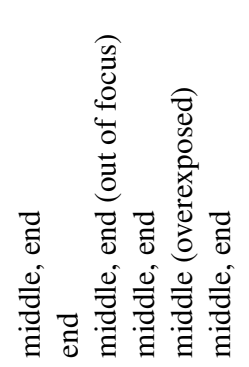 & 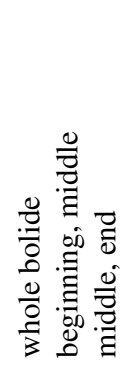 & 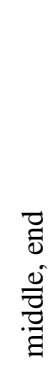 \\
\hline 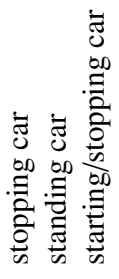 & 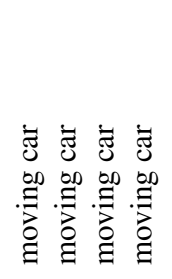 & $\begin{array}{l}\text { है } \\
00 \\
.5 \\
0 \\
0 \\
\Xi\end{array}$ & $\stackrel{\nexists}{:}$ & 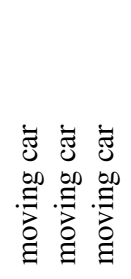 & 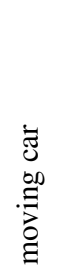 & 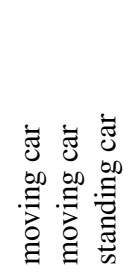 & 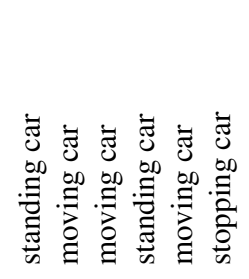 & 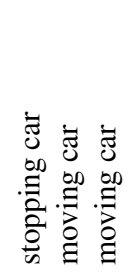 & 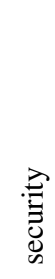 \\
\hline 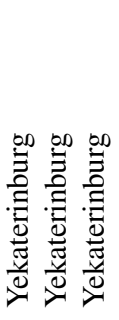 & 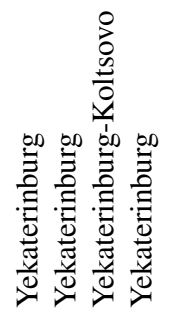 & 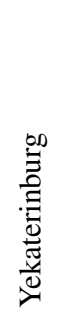 & 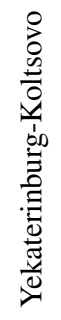 & 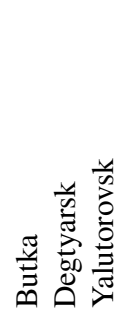 & 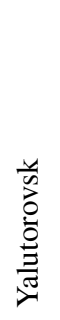 & 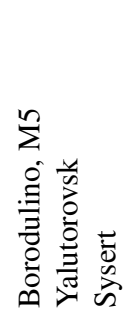 & 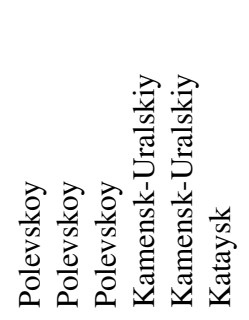 & 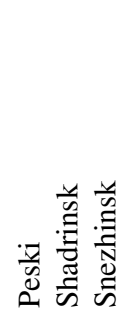 & 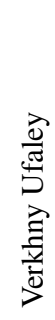 \\
\hline 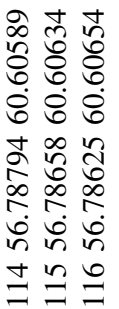 & 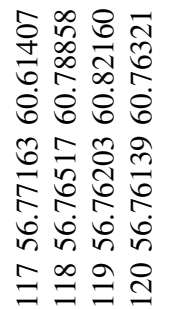 & 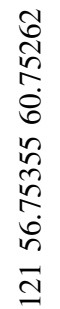 & 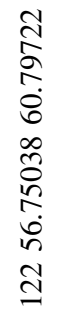 & 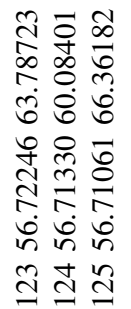 & 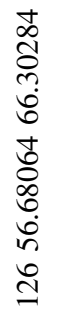 & 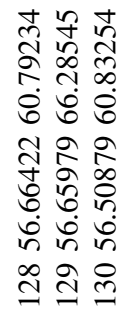 & 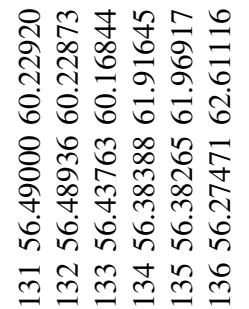 & 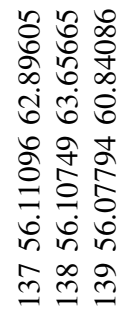 & 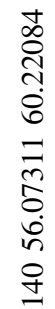 \\
\hline
\end{tabular}




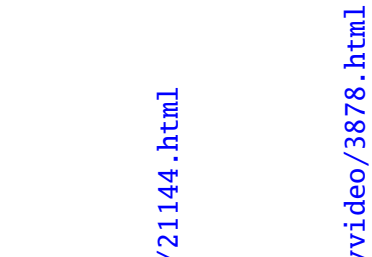

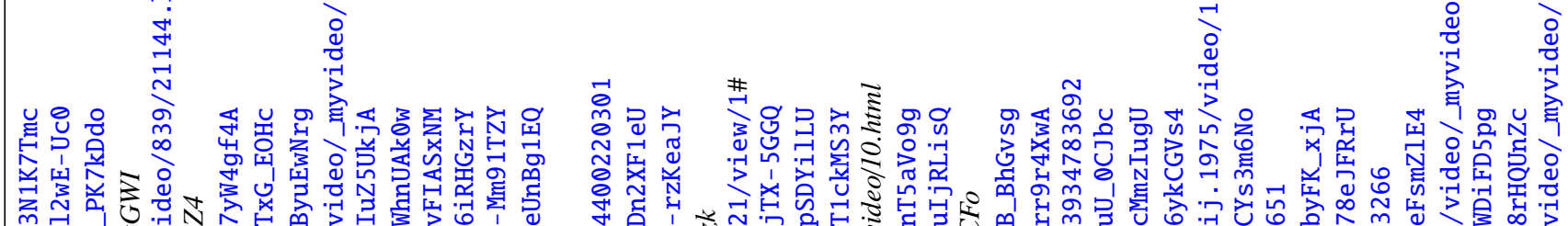

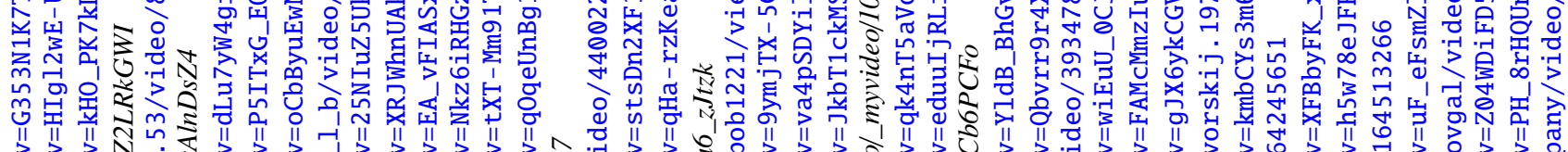

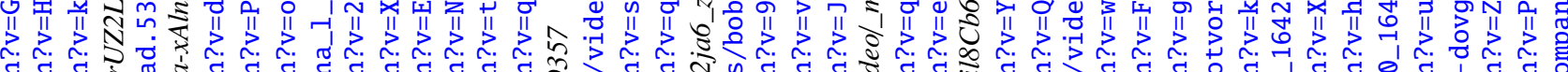
yु $y$ yु

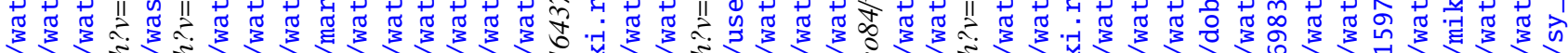

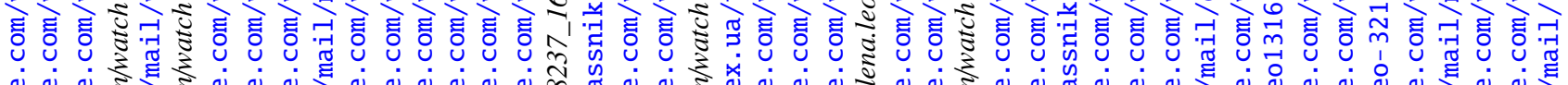
ه̊

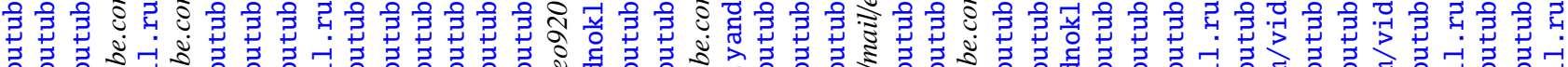

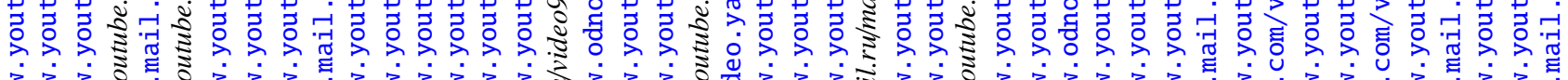

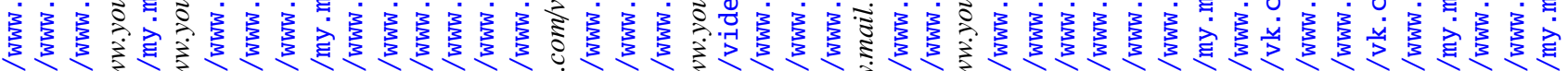

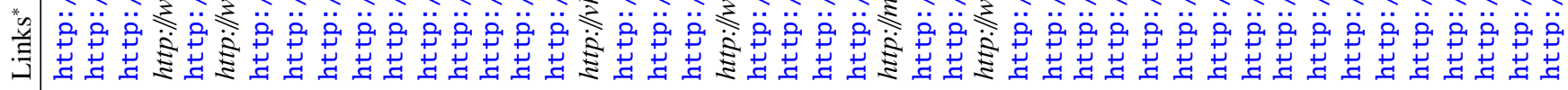

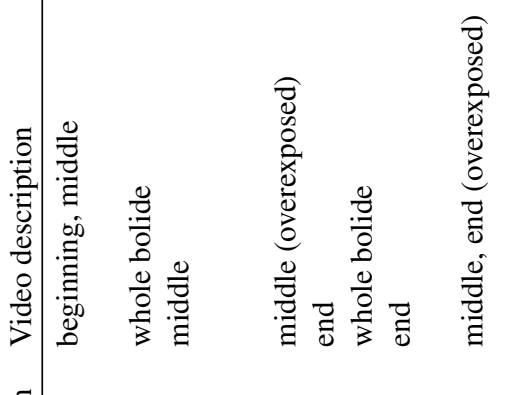

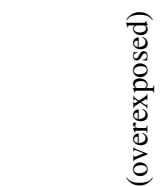

.ี

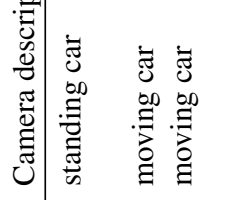

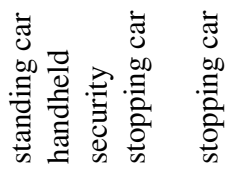

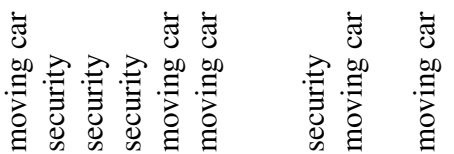

ซ 竞:

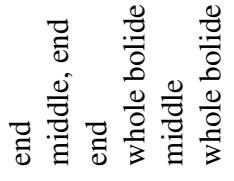

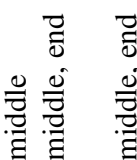

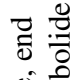
$\frac{0}{\frac{0}{3}} \frac{0}{3}$

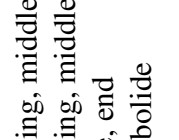

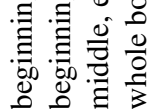

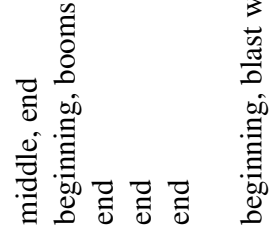

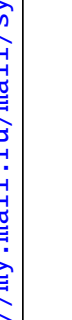


J. Borovička et al.: A catalog of video records of the 2013 Chelyabinsk superbolide

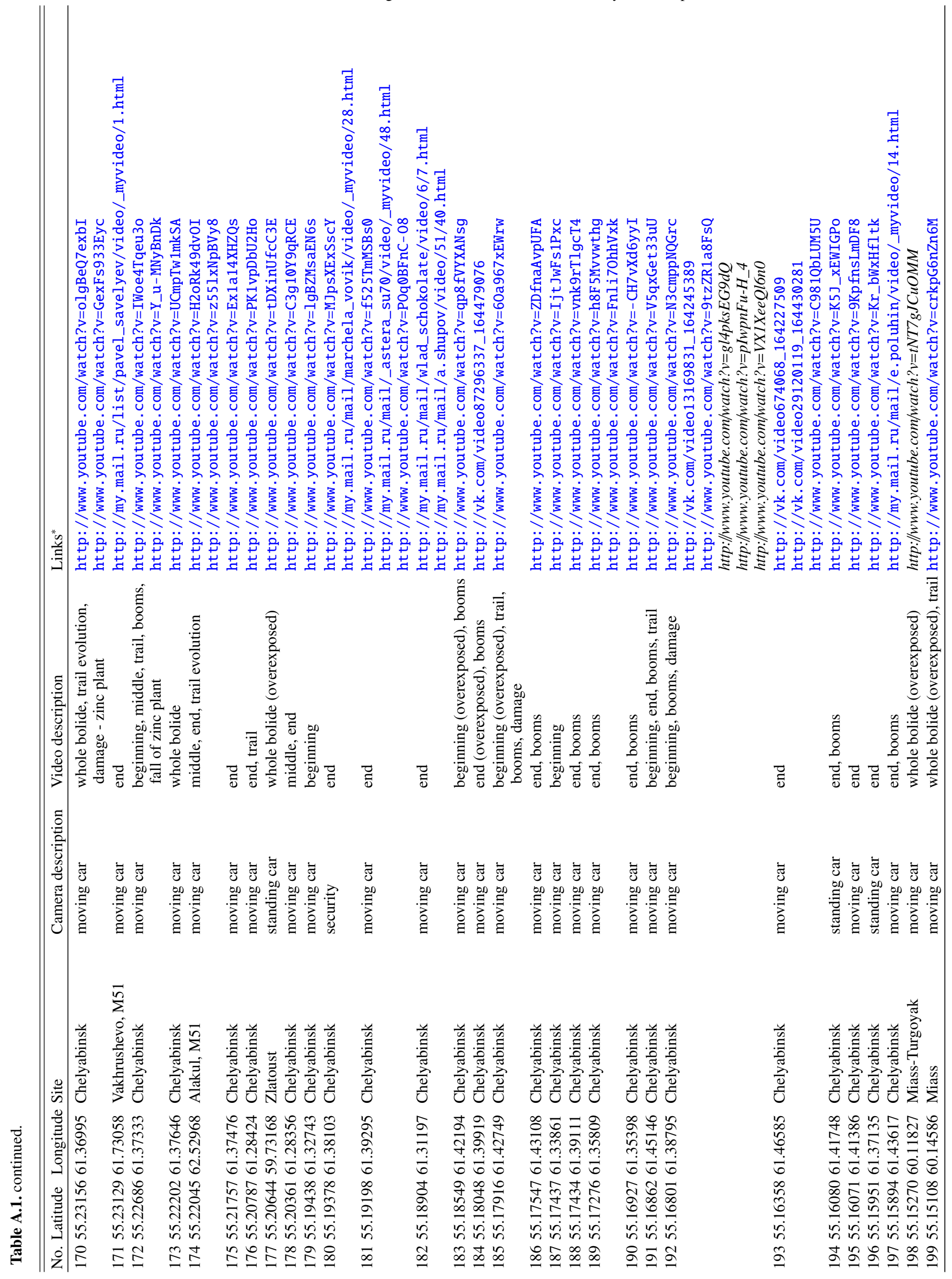


A\&A 585, A90 (2016)

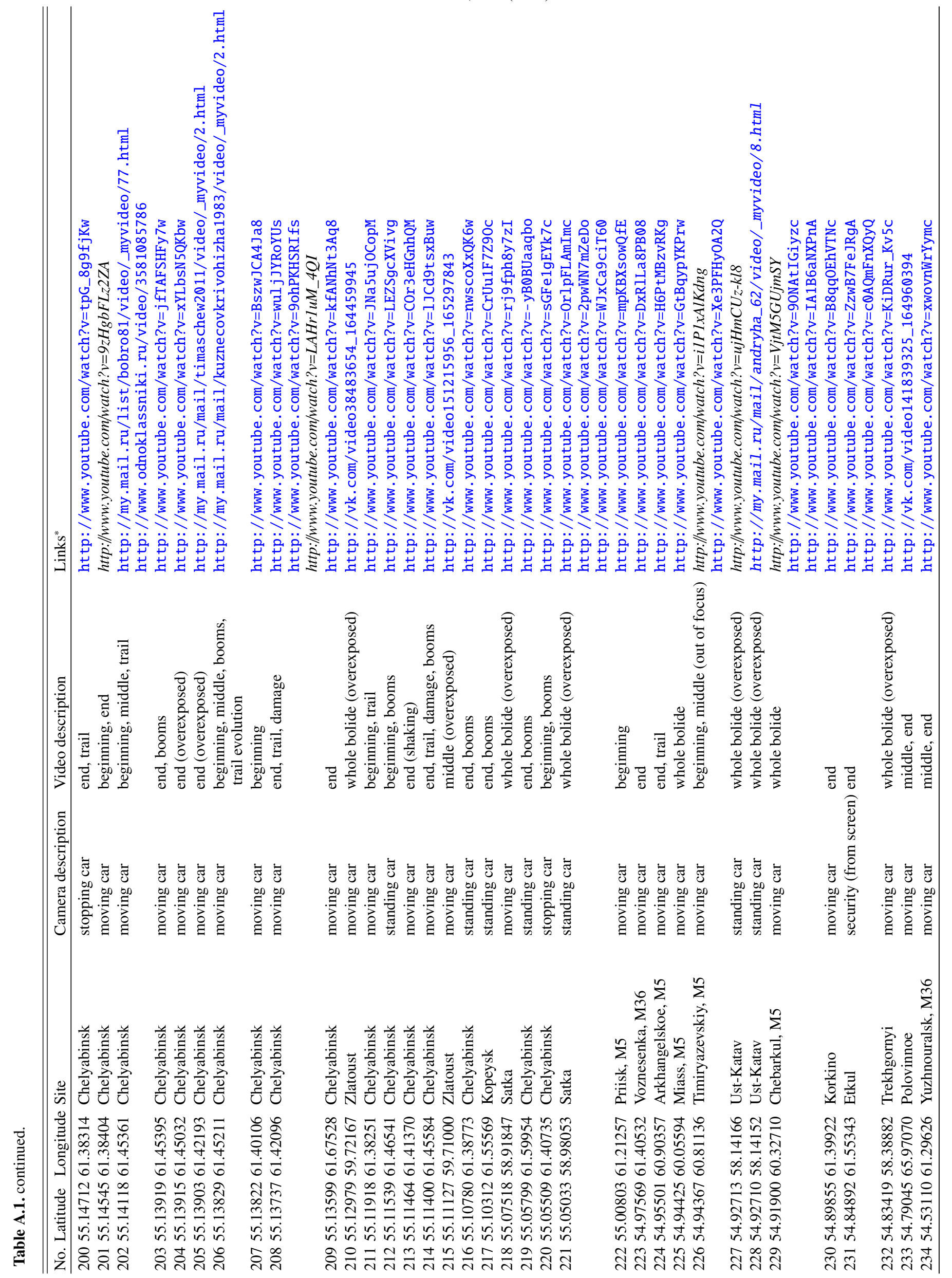


J. Borovička et al.: A catalog of video records of the 2013 Chelyabinsk superbolide

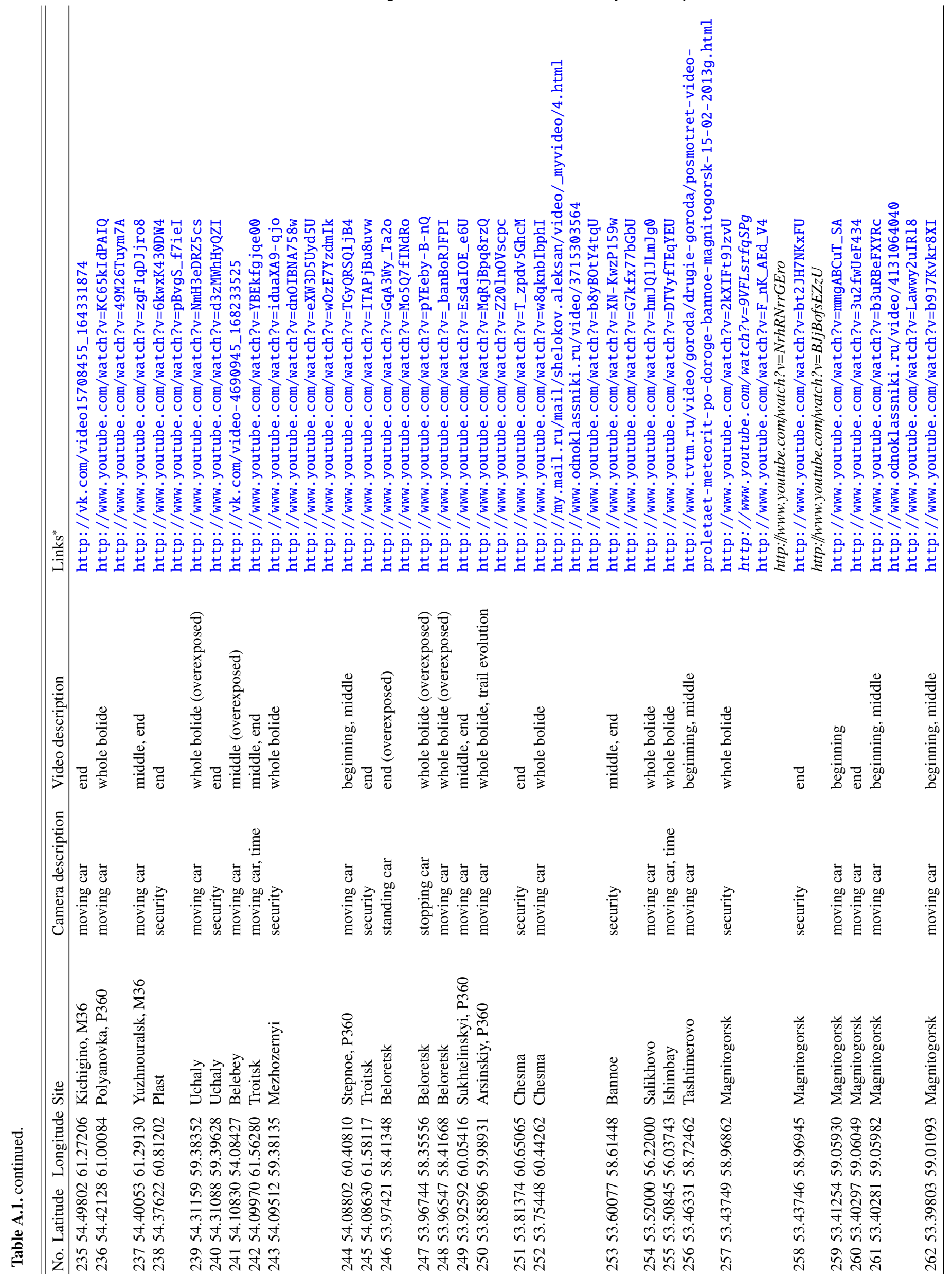


A\&A 585, A90 (2016)

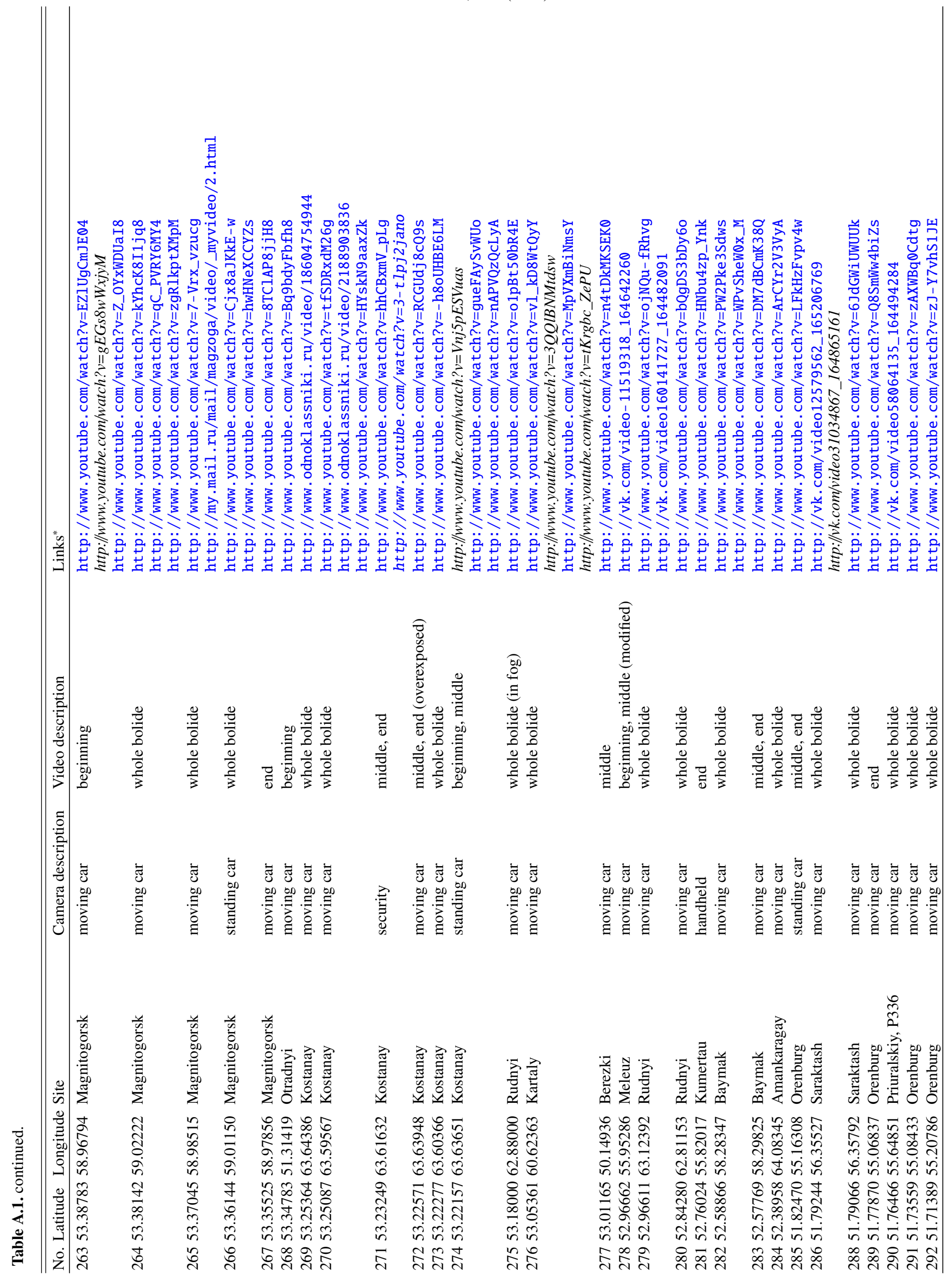


J. Borovička et al.: A catalog of video records of the 2013 Chelyabinsk superbolide

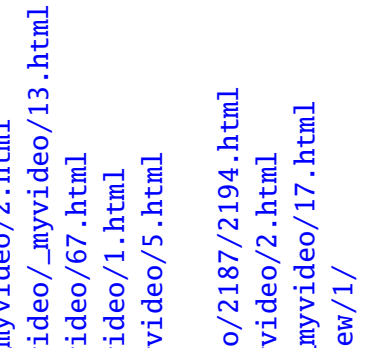

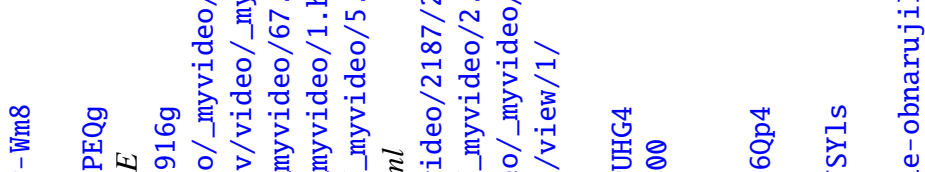

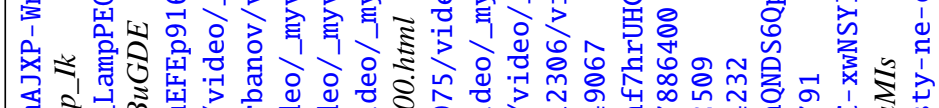

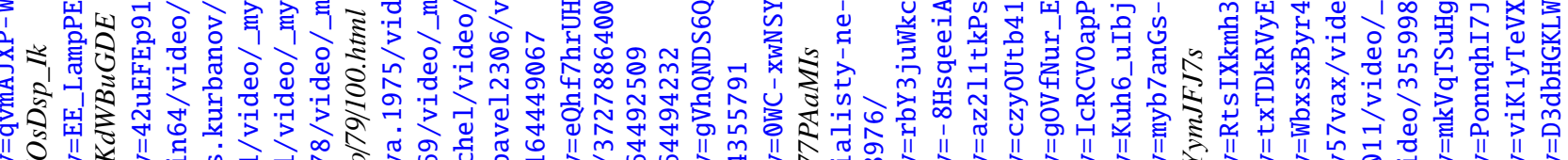

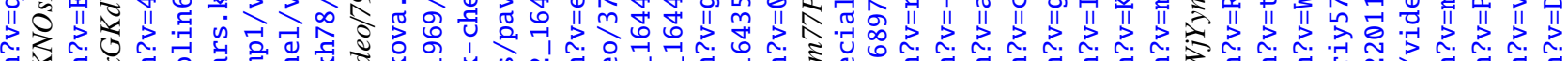

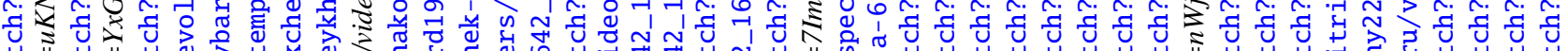
至 引 ¿ं

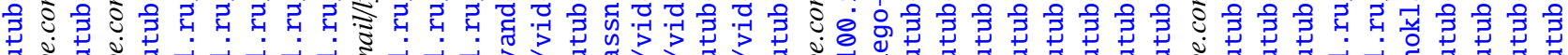

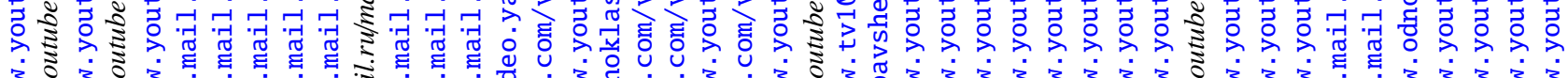

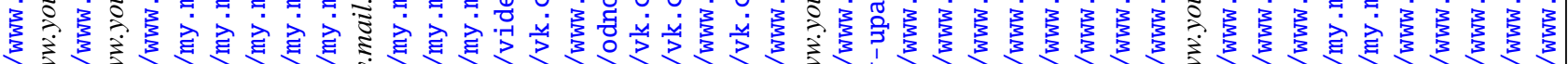

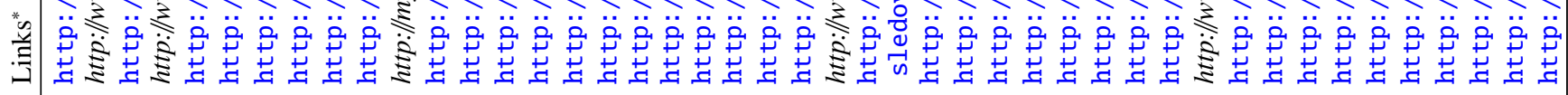

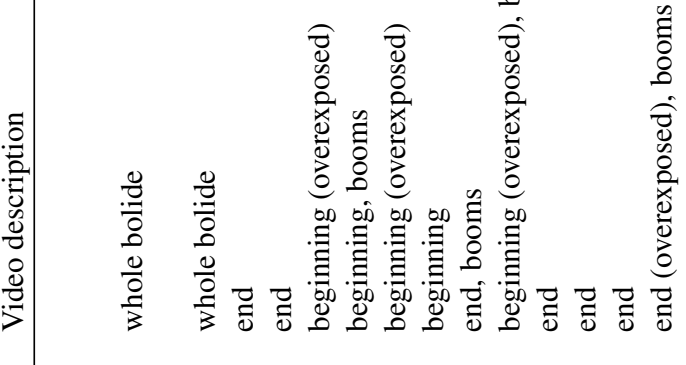

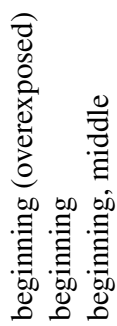

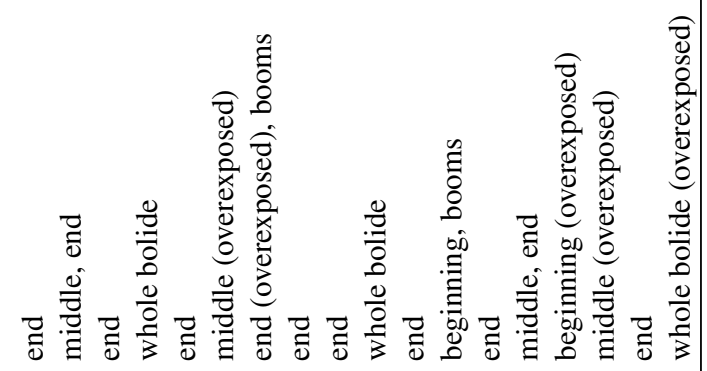

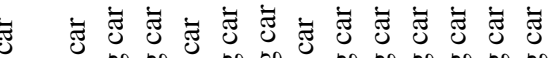

పี ฮี ซี

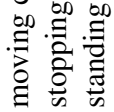

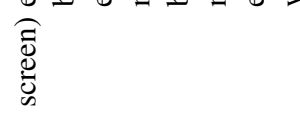




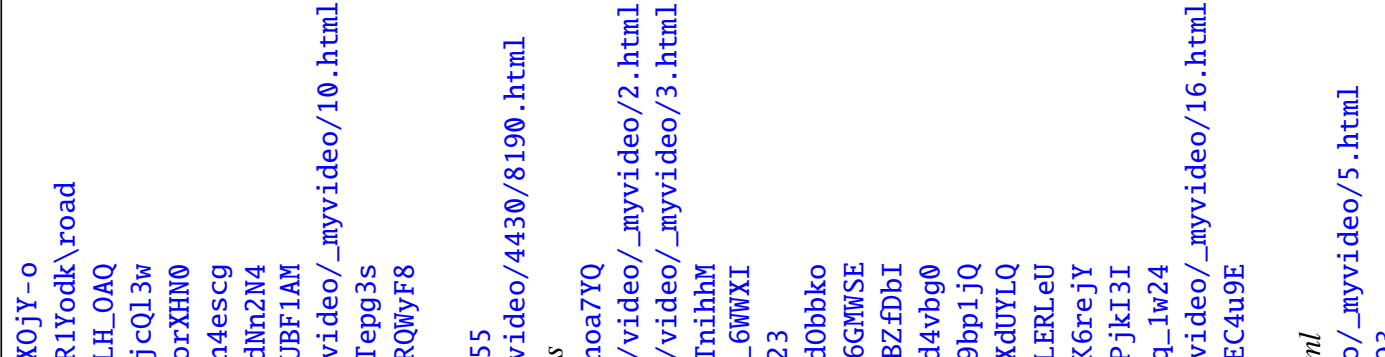

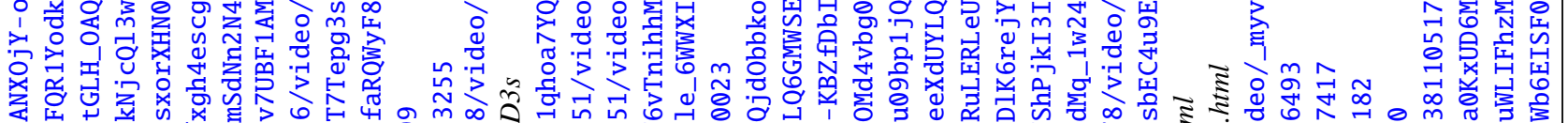

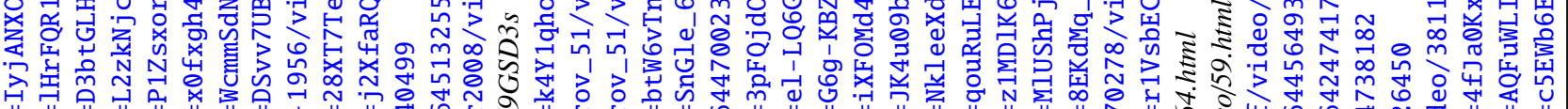
II II $\|$ II $\|$ II II I II II

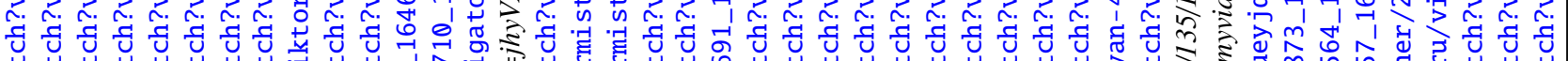

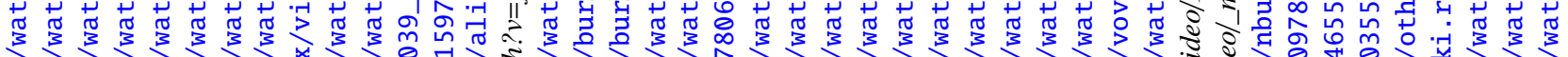

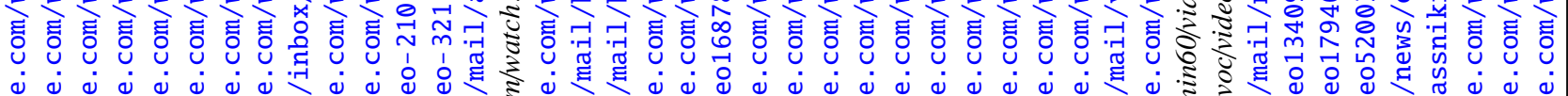

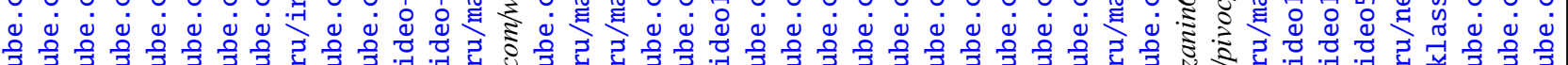

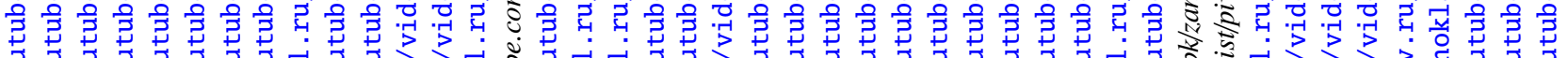

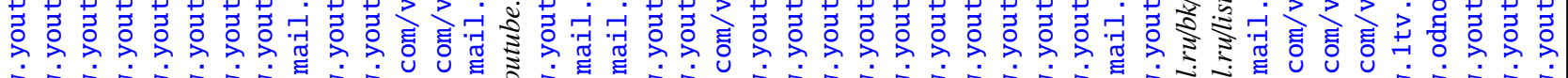

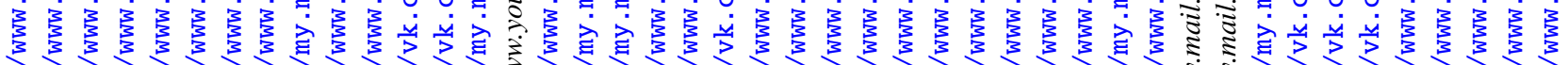

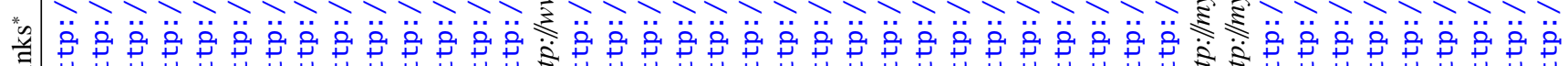

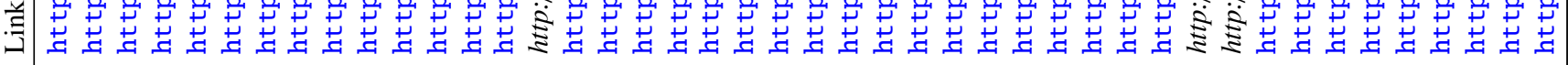<smiles>C=CC(C)CCC</smiles>

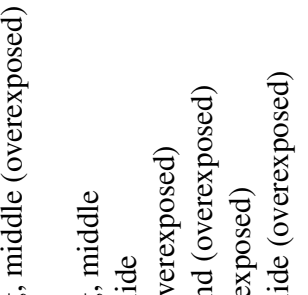

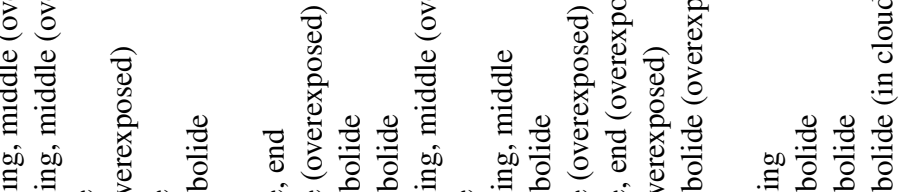

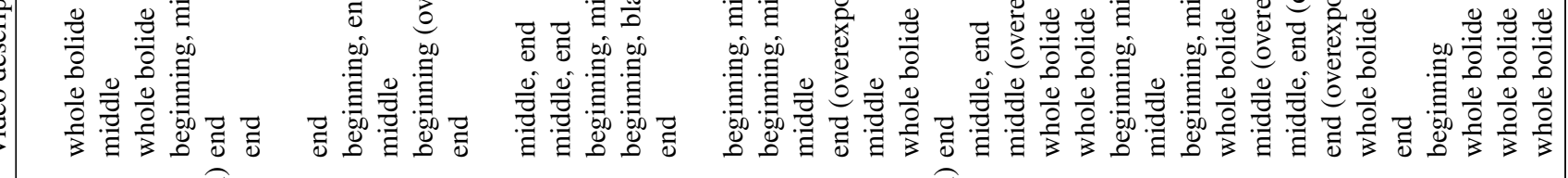

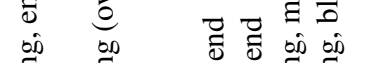

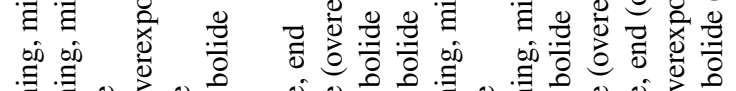
: ญ्ञ̄

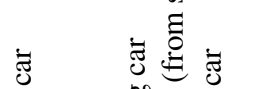

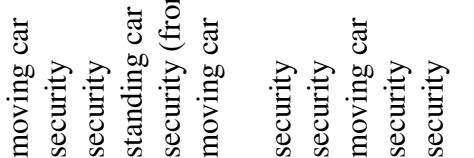
ซี ซี

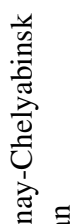

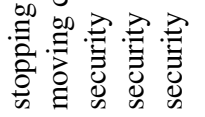

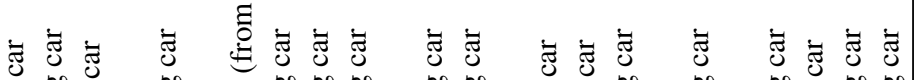
厄్

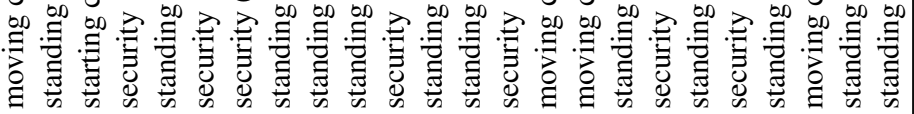

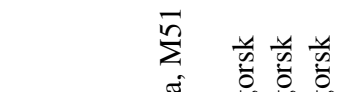

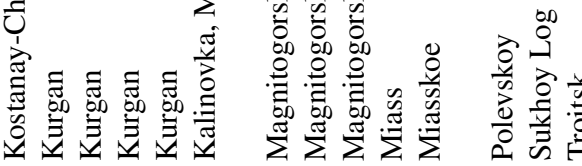

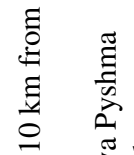

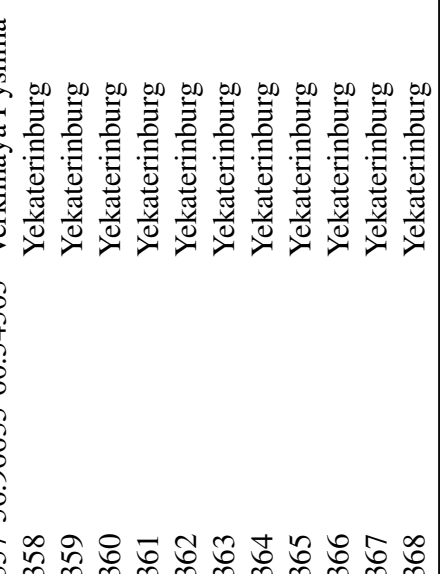

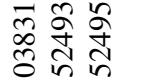

กู่

요

ชิ송

它语

ते ले ले ले ले ñ ñ nin

ले ले ले ले mे

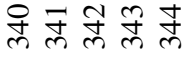

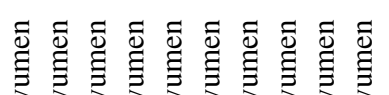

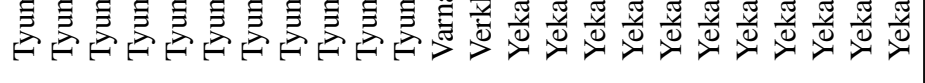

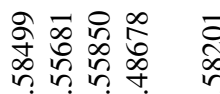

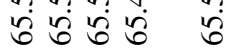

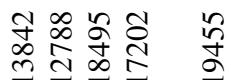

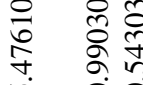

ช่ 88

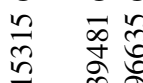

in in in in in in

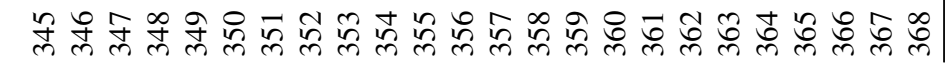


J. Borovička et al.: A catalog of video records of the 2013 Chelyabinsk superbolide

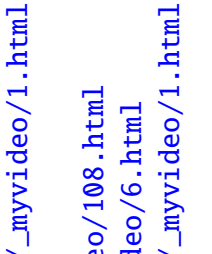

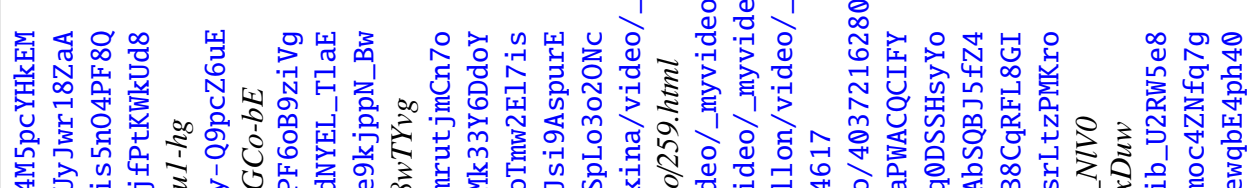

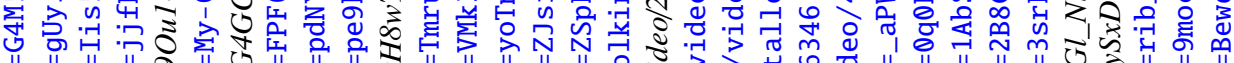

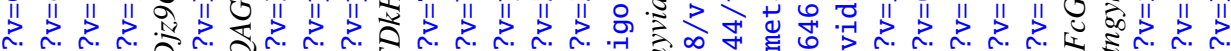

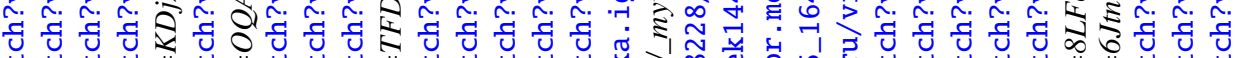

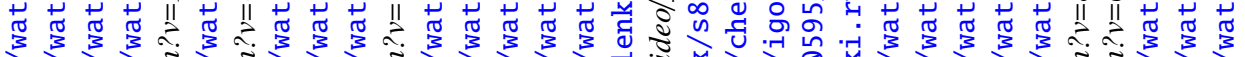

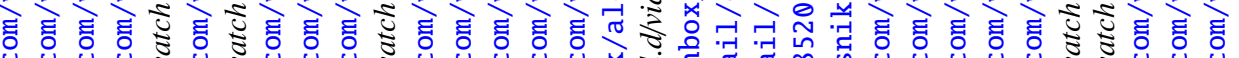

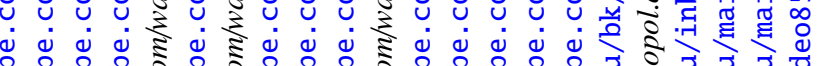

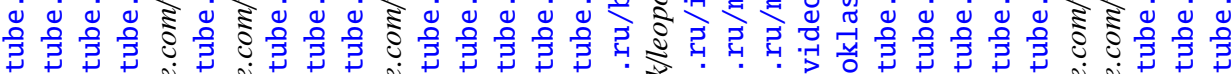

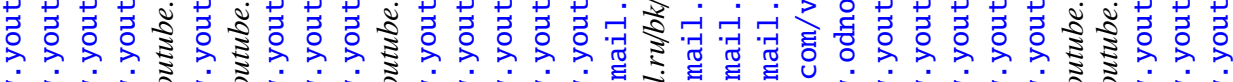

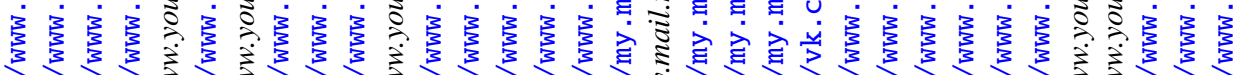

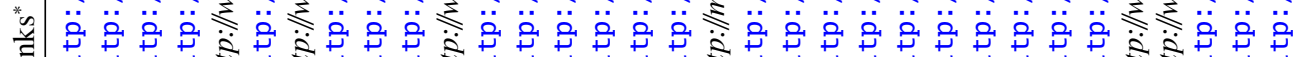

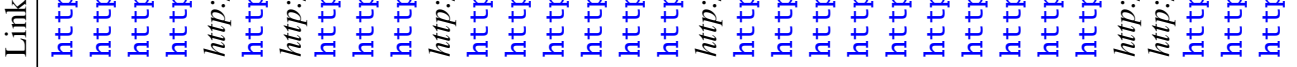

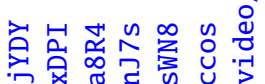

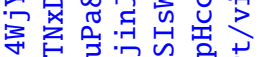

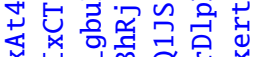

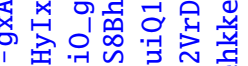

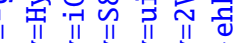

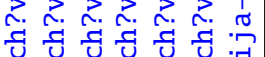

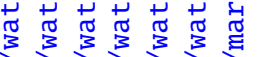

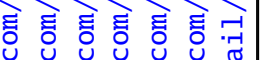

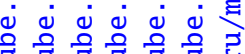
宩

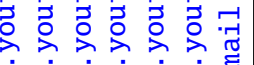

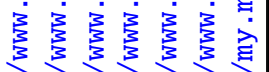

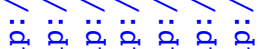

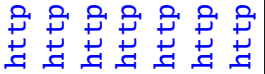

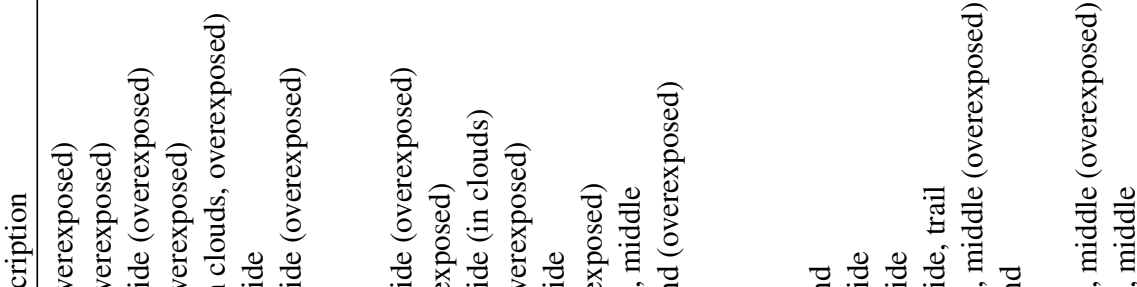
:气

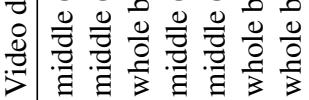
: 용 요 छ

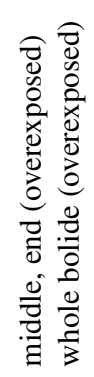

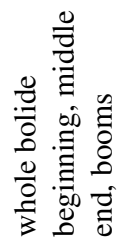
क्षे

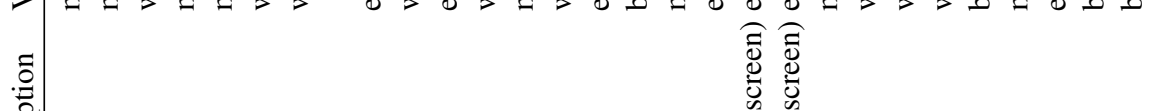

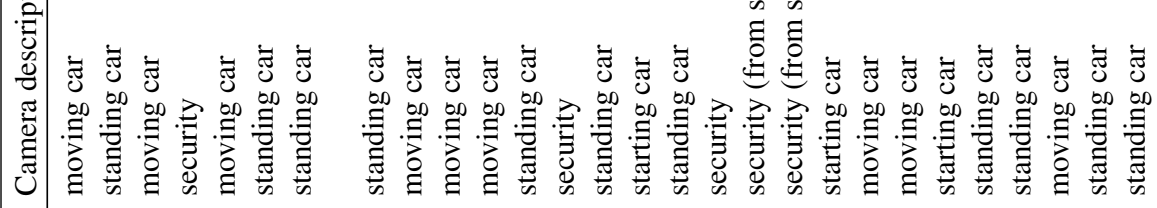

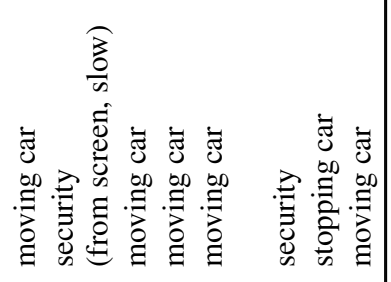

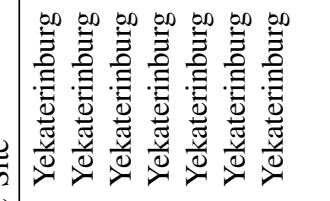

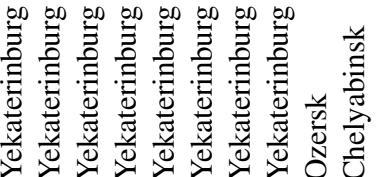

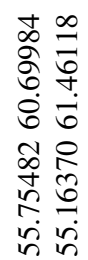

:
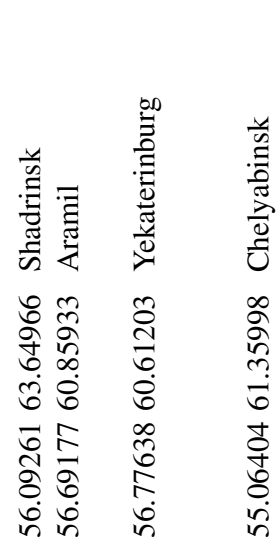
in in

ఫे के के

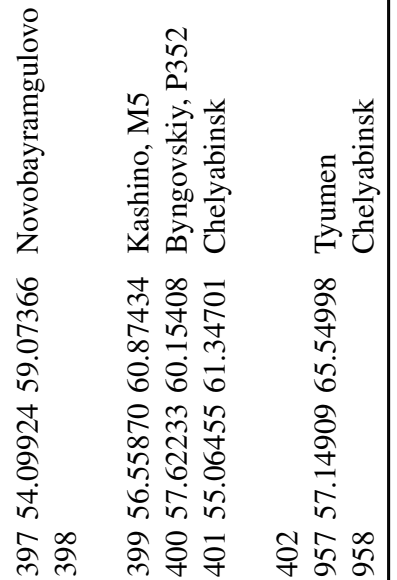

A90, page 19 of 35 


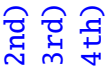

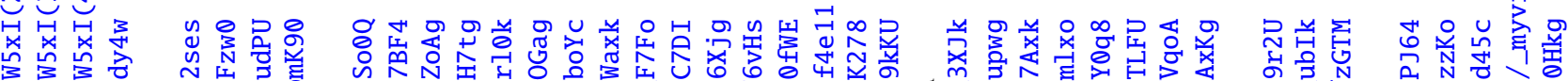

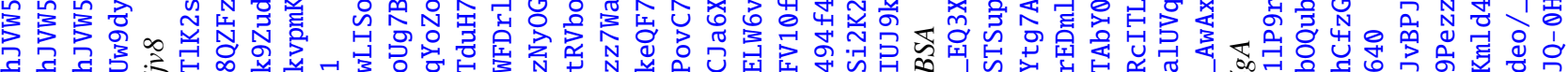

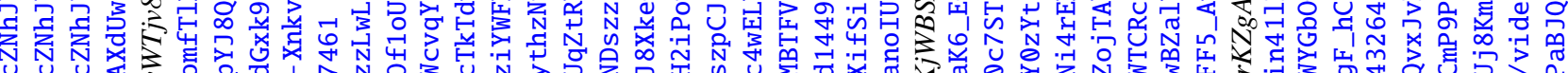

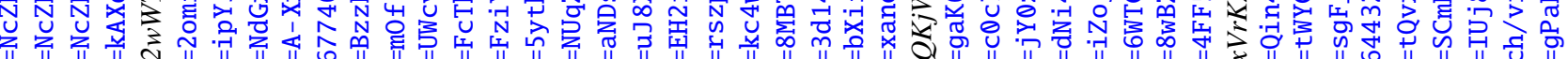

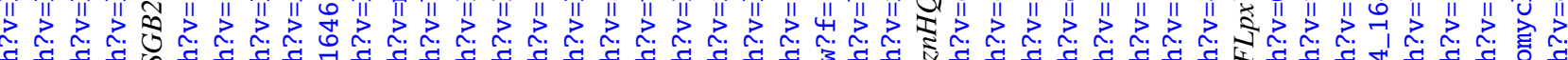

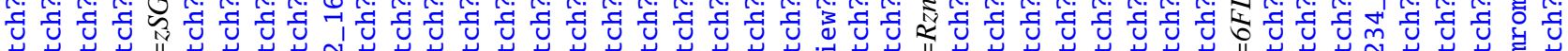

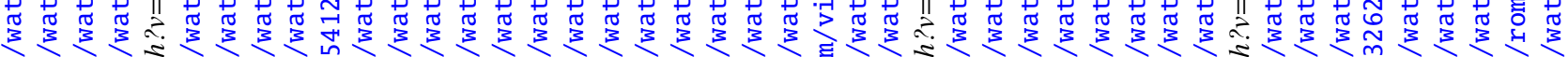

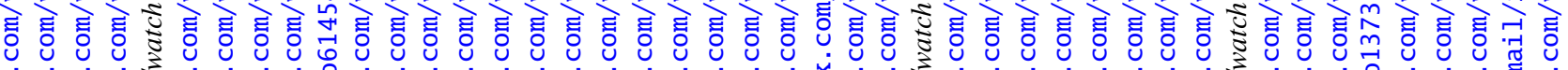

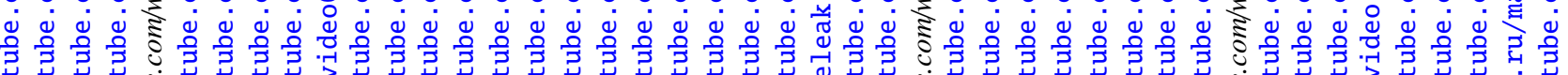

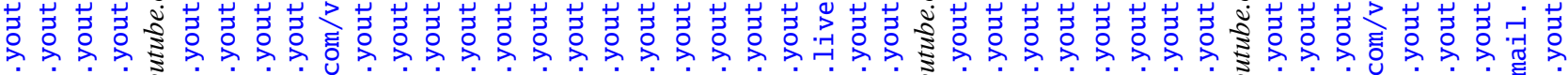

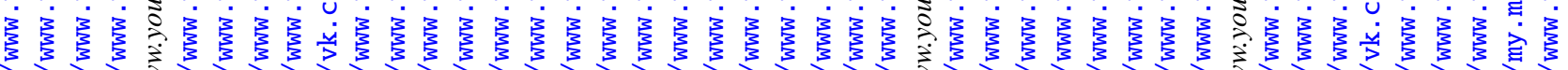
茬

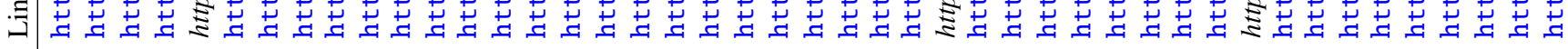

:

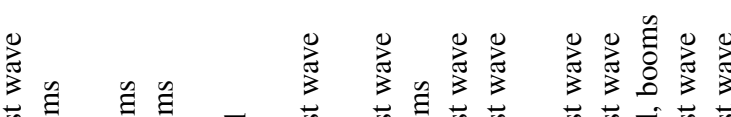

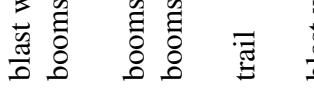

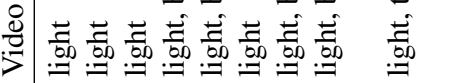

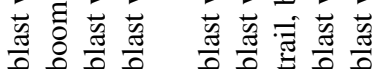

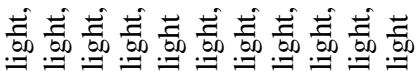

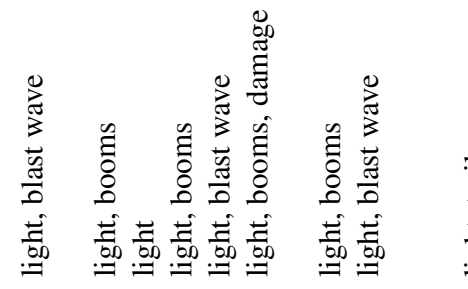

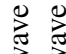

蛋莺

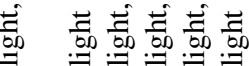

:

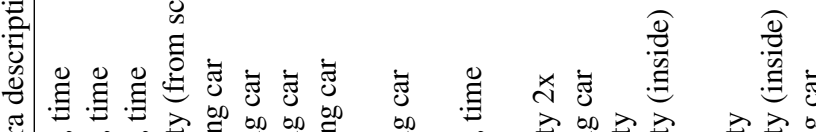

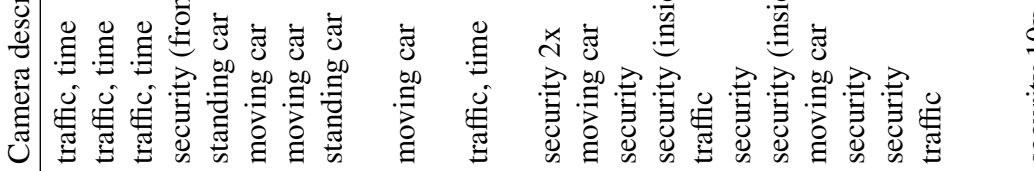
:

ซี ซี ซี ซี

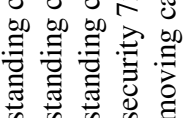

竎

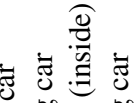

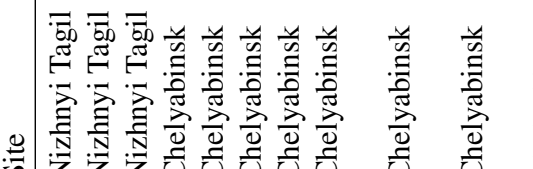

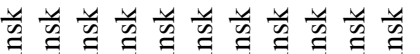

:

$\ddot{y} \ddot{y} \ddot{y} \ddot{m}$

㟧泀

$\pi \pi$

蛋

ग

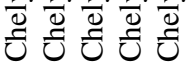

过

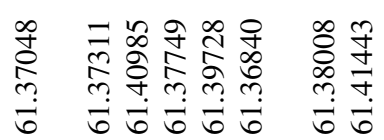

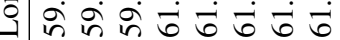

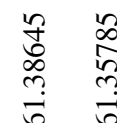

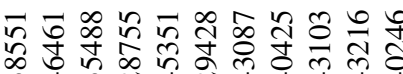

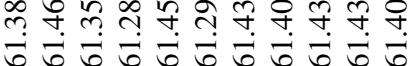

के 5060

- $6 \overrightarrow{6} \overrightarrow{6} 8$

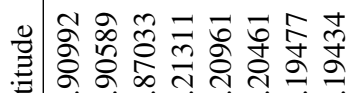

\&

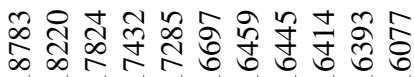

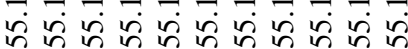

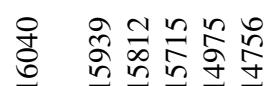

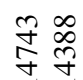

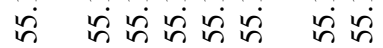

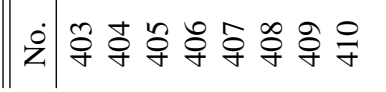

‡

获 


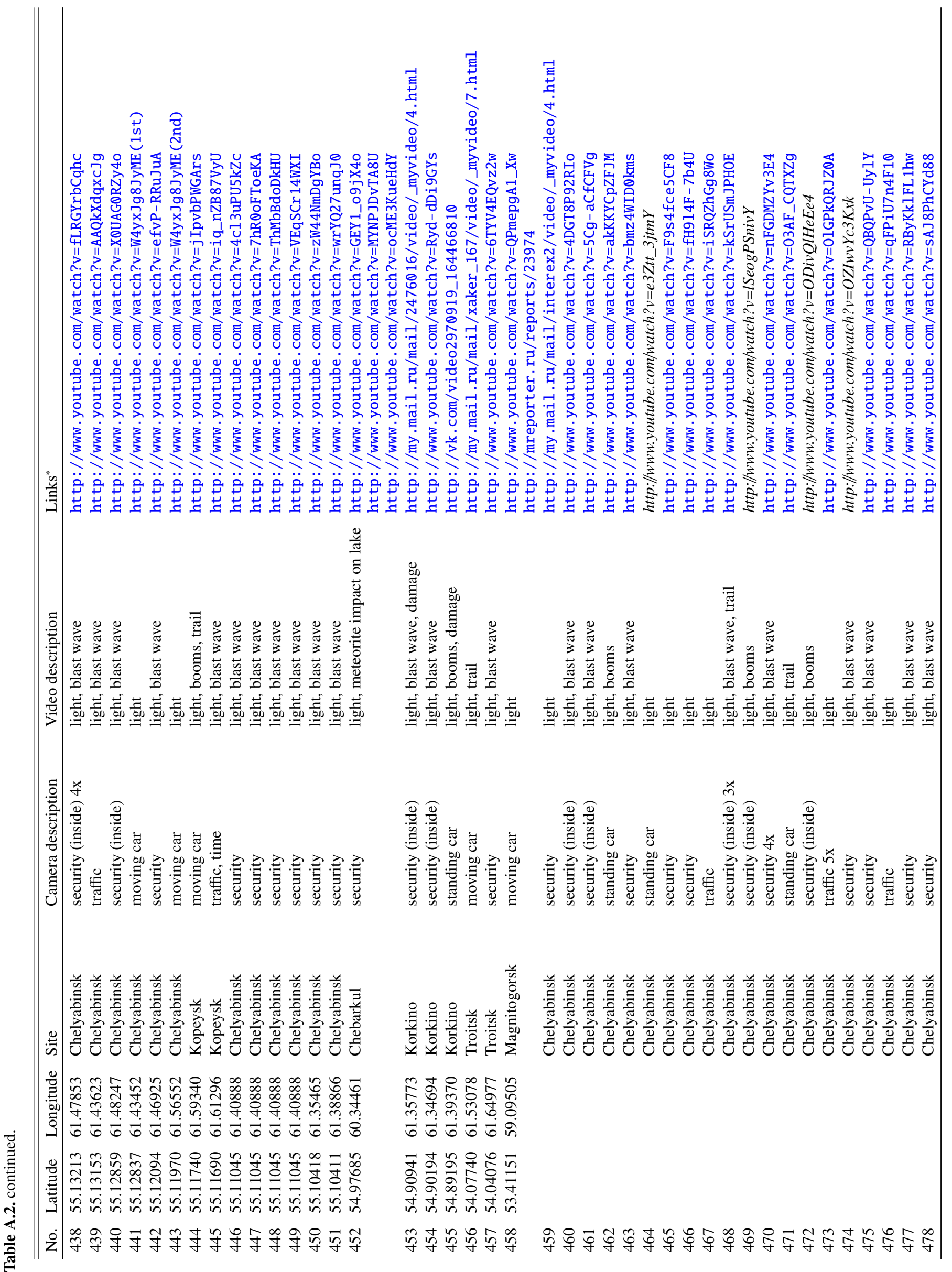




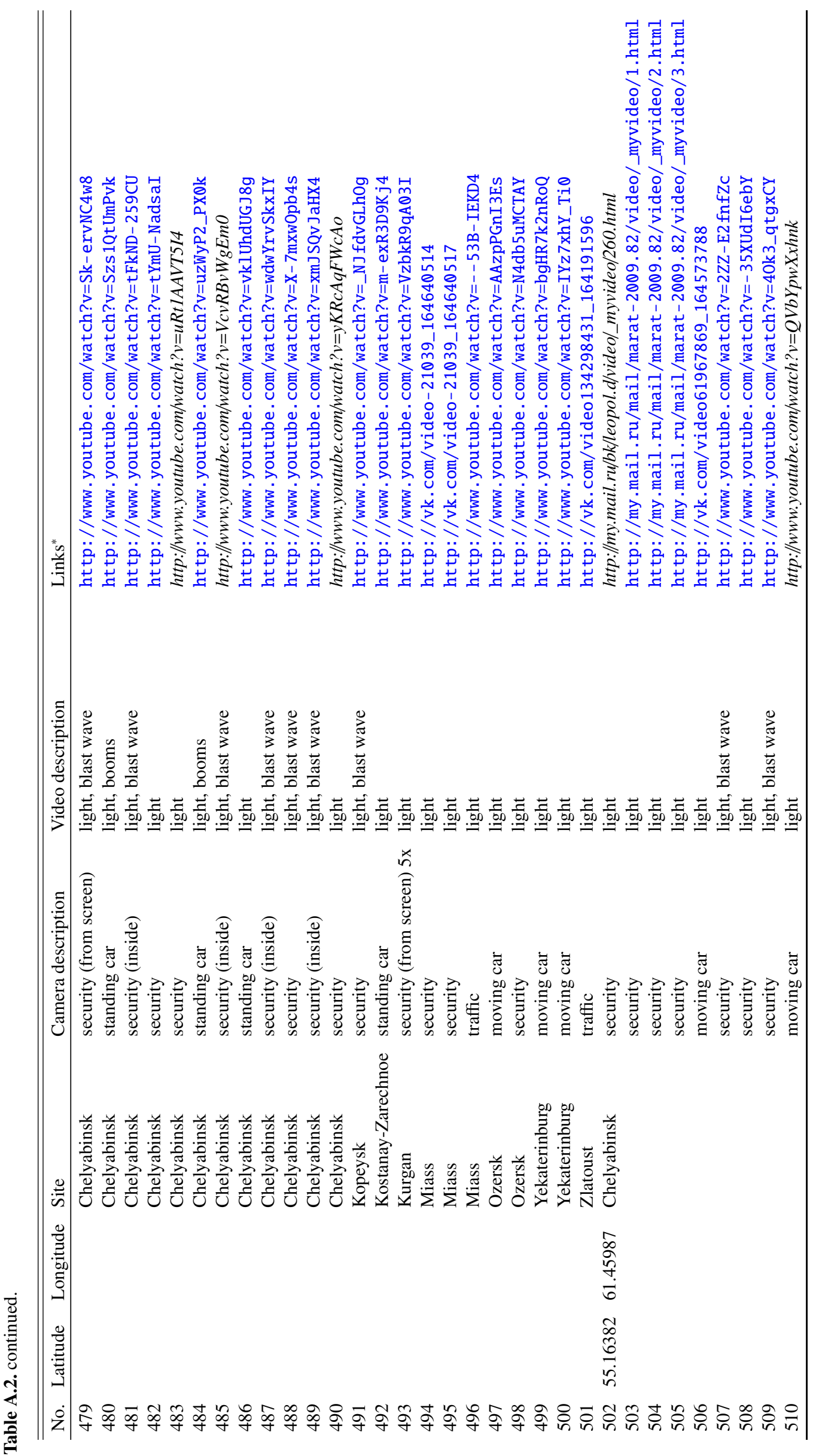




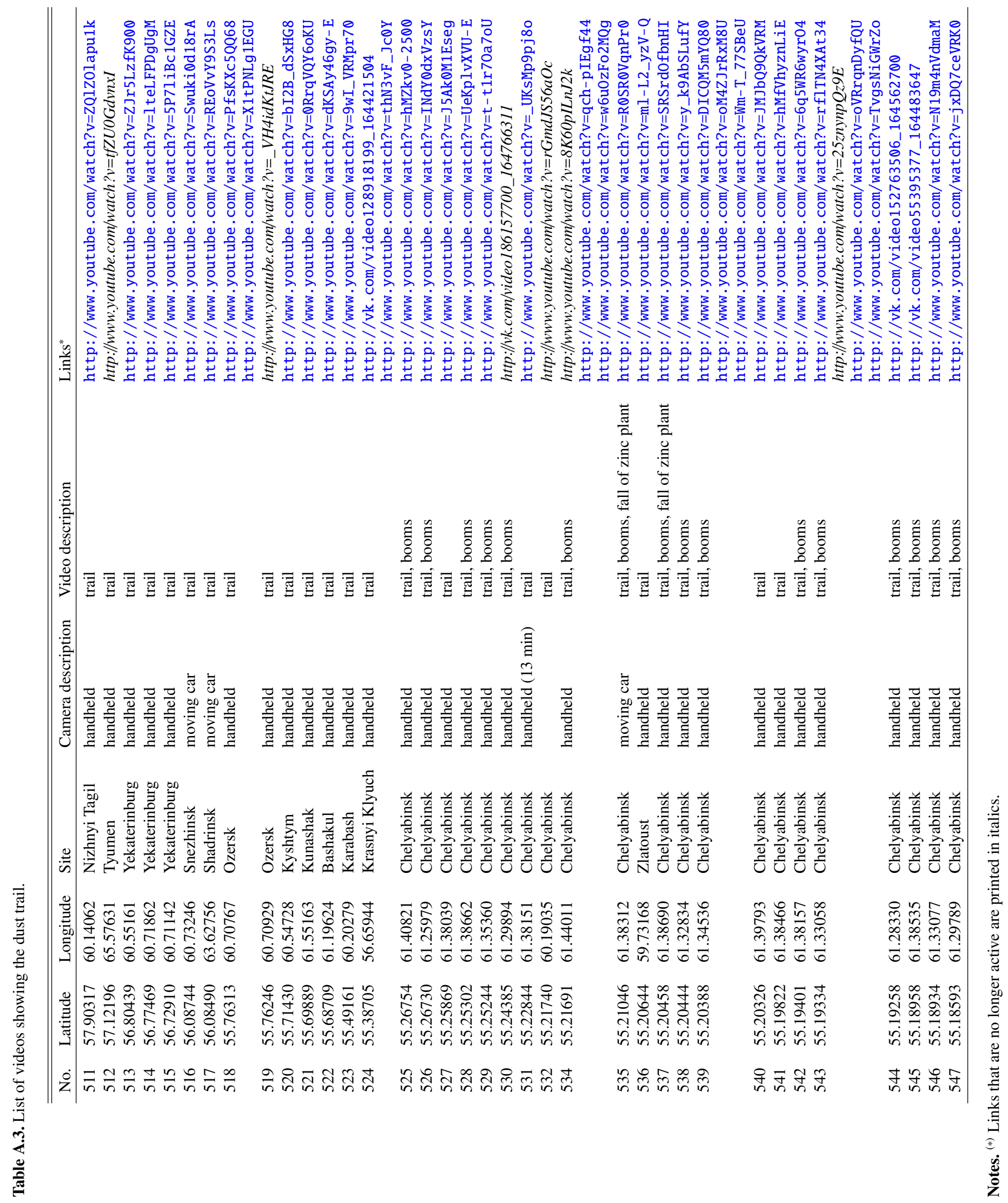




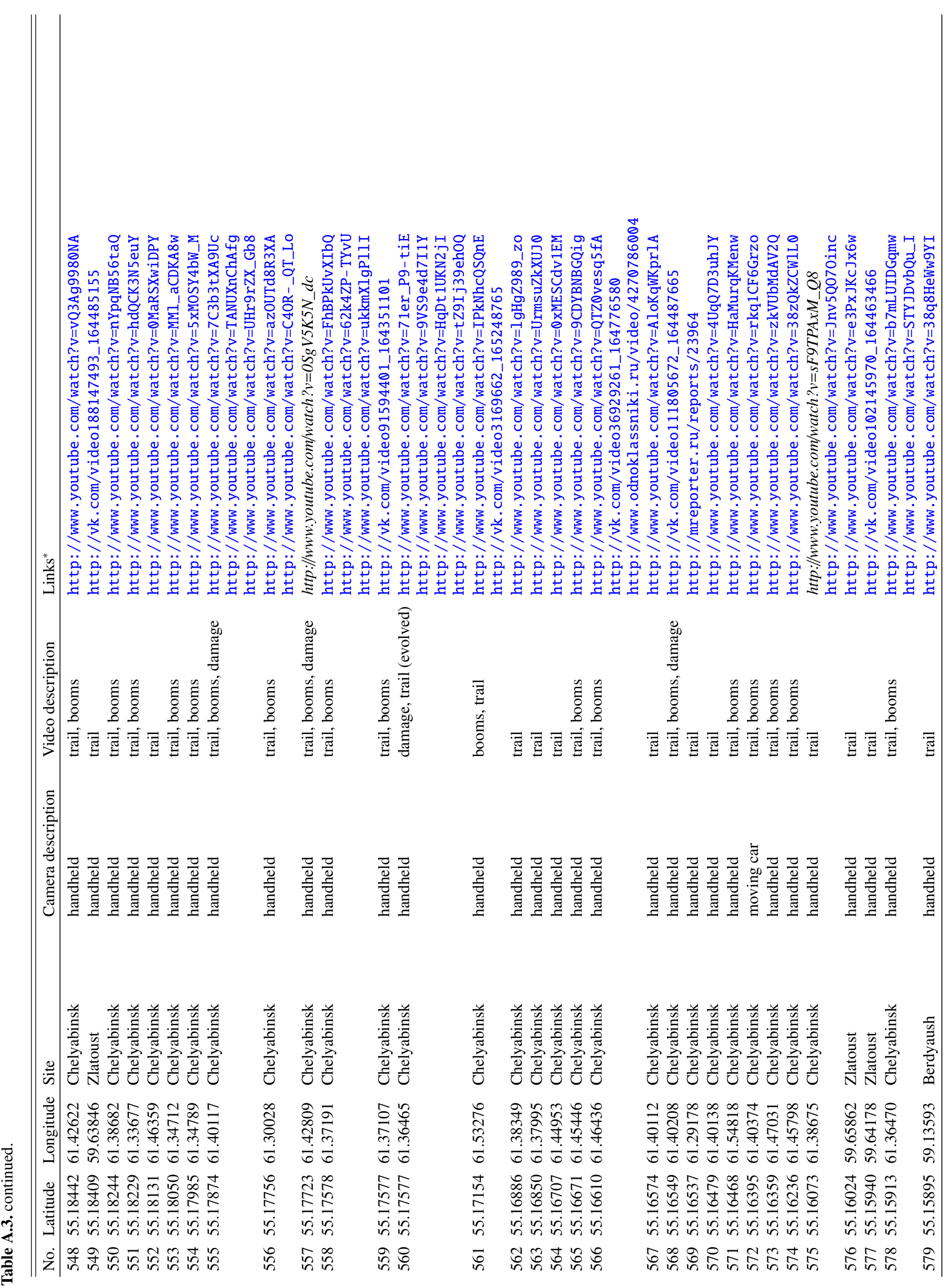


J. Borovička et al.: A catalog of video records of the 2013 Chelyabinsk superbolide

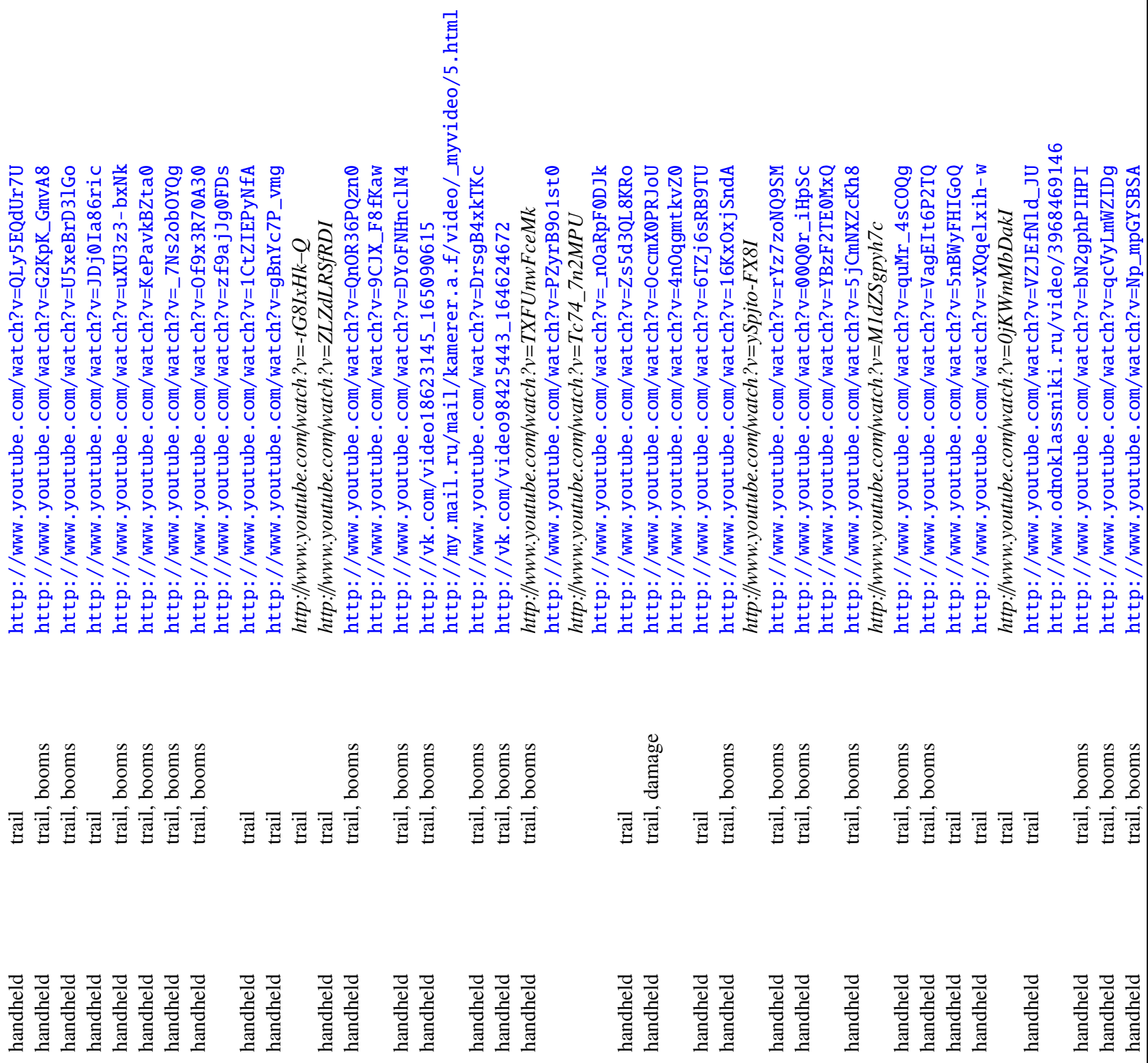

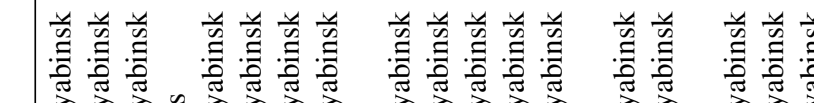

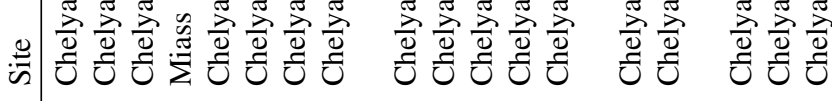

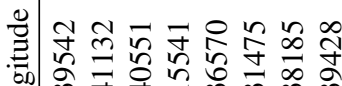

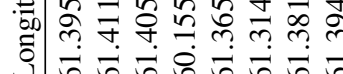

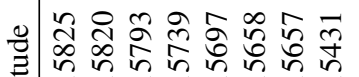

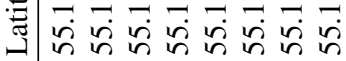

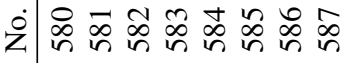

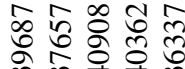

mे mे 워

$6 \overrightarrow{6} \dot{6} \overrightarrow{0}$

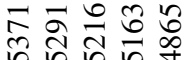

两 $=7$

$\infty$ वे चर

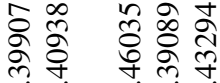

उ० $6 \dot{6} 6$

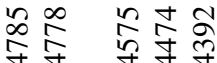

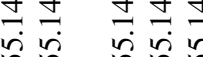

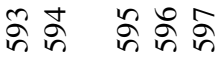

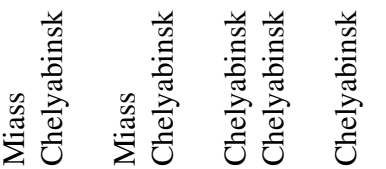

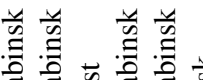

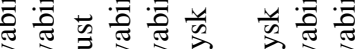

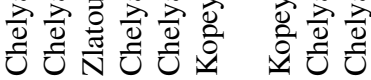

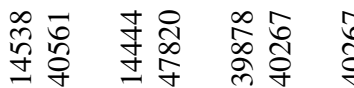

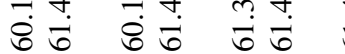

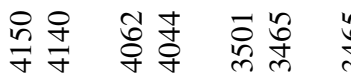

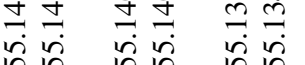

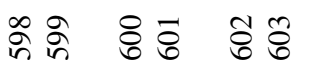

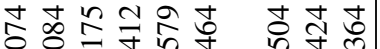

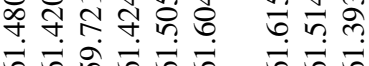

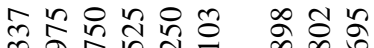

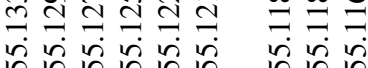

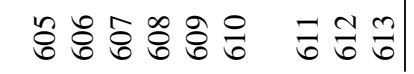




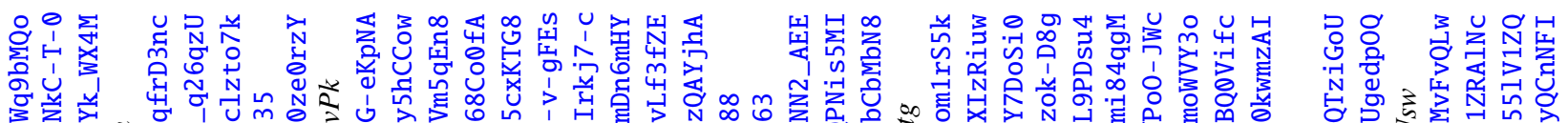

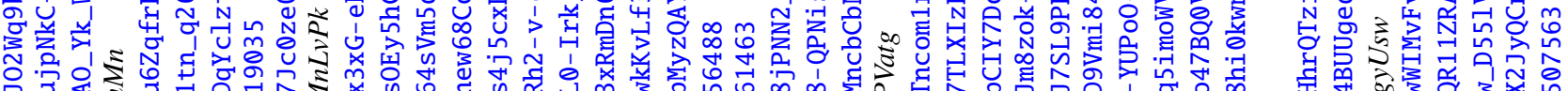

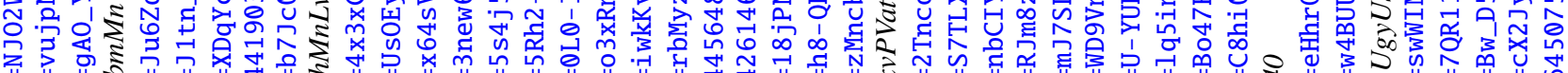

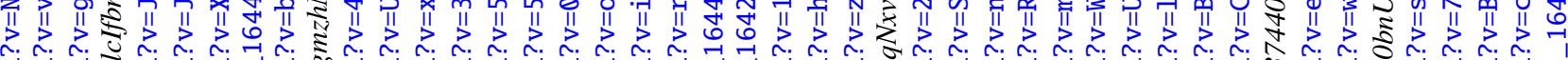

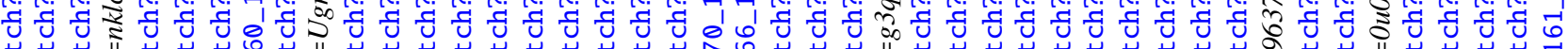

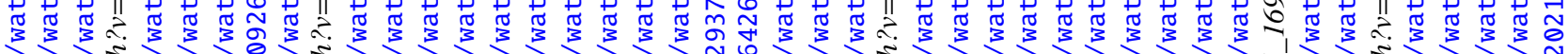

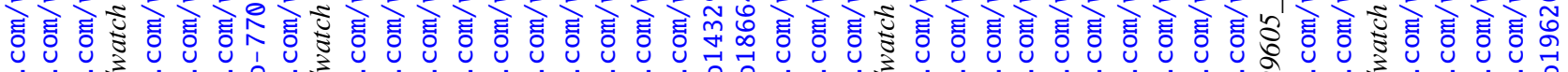

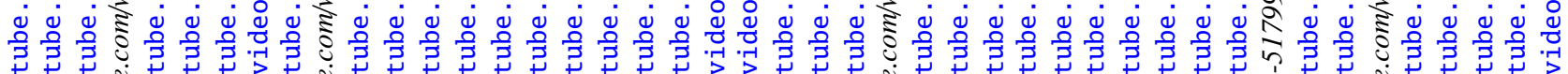

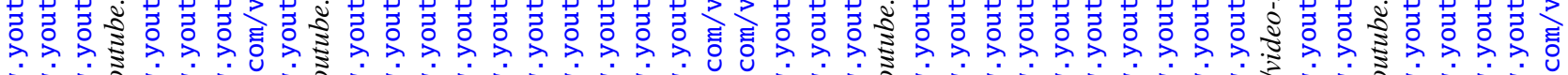

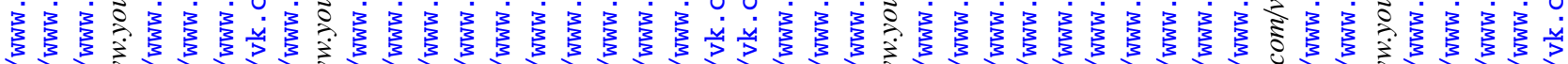

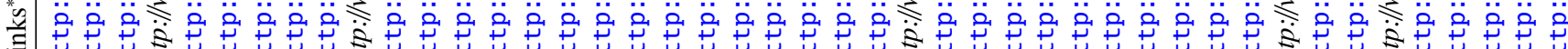

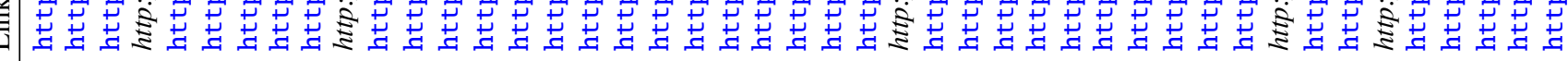

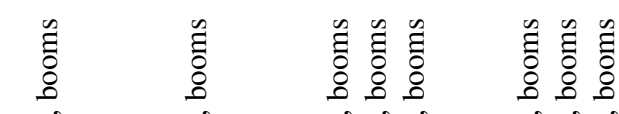

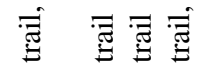

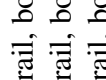

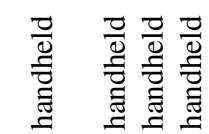

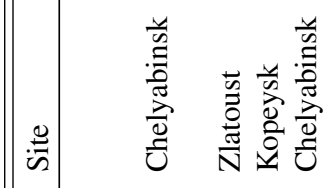

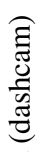

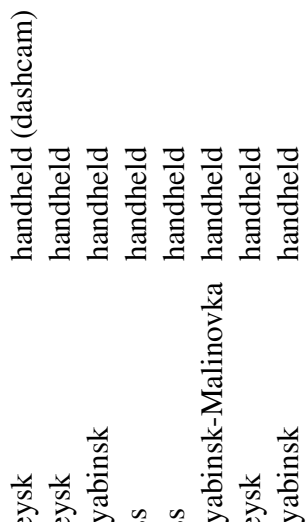

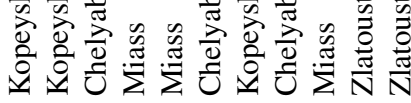

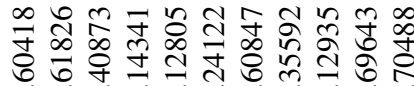

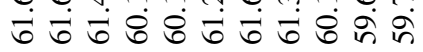

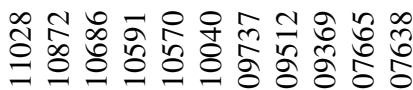

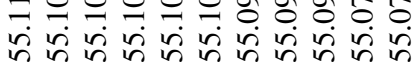

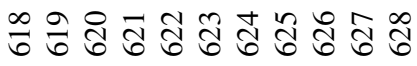

है है

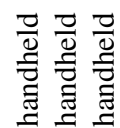

뮴

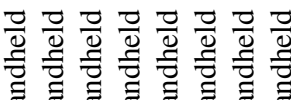

뮴<smiles>C1CCC1</smiles>

ถั

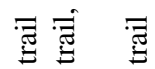

竞

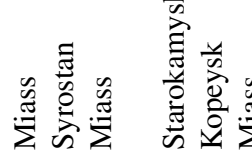

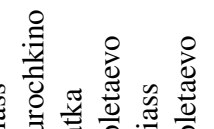

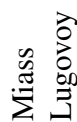

急命尌

0

ธี่

in

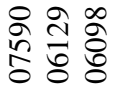

in

च융응

in in

ते

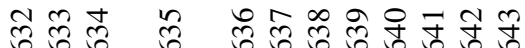

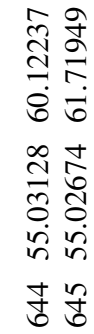




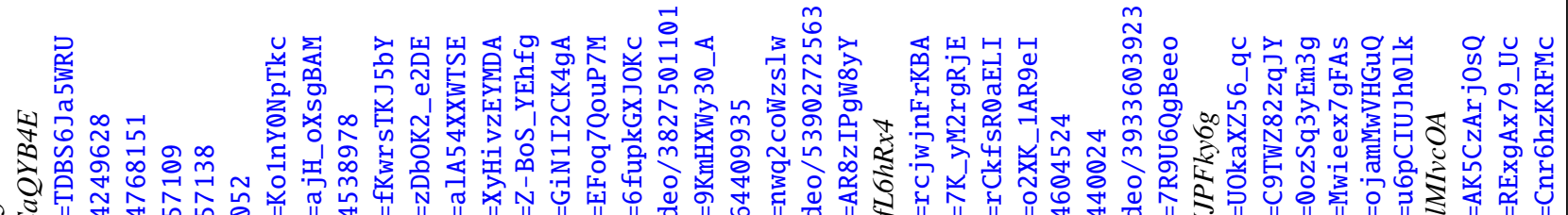

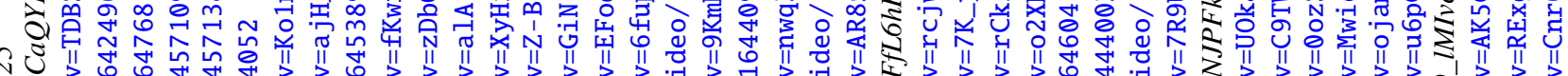

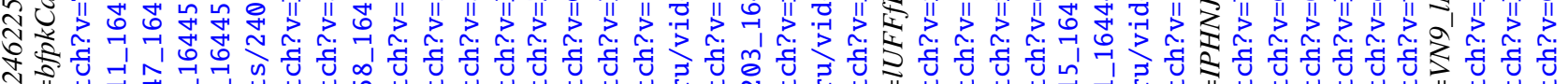

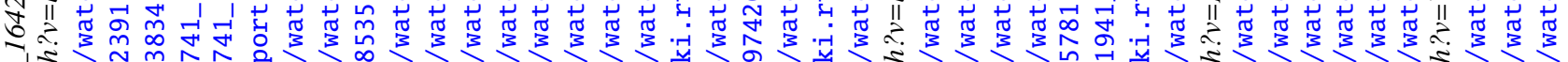

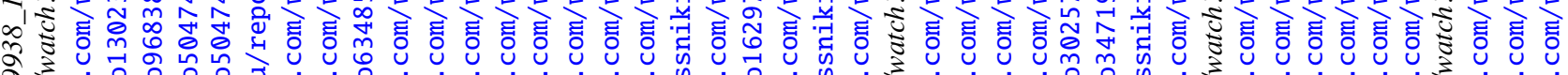

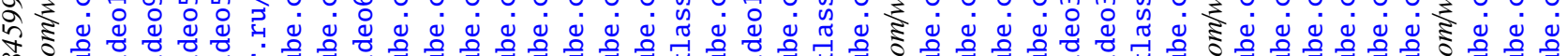

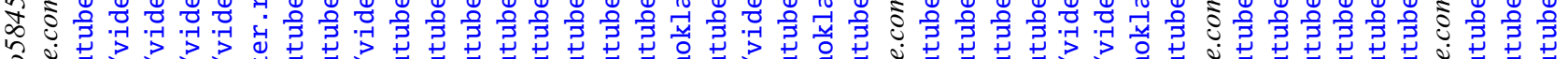

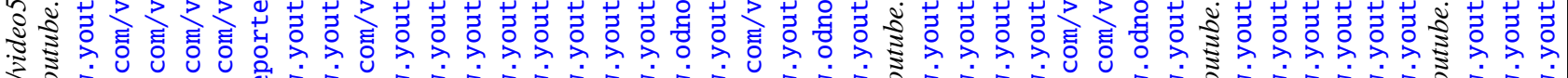

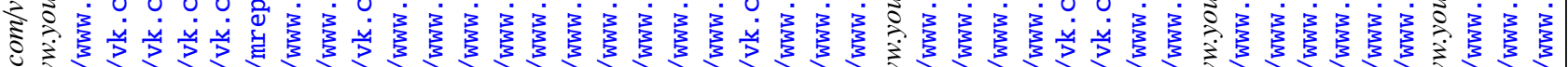

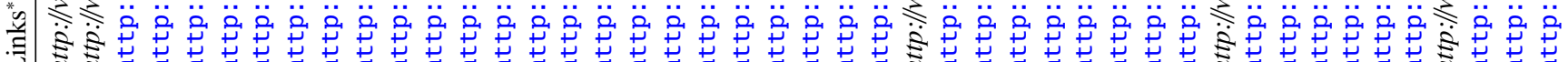

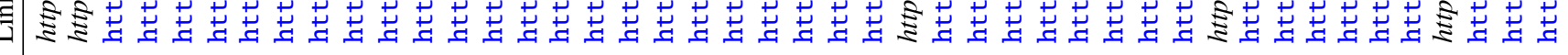

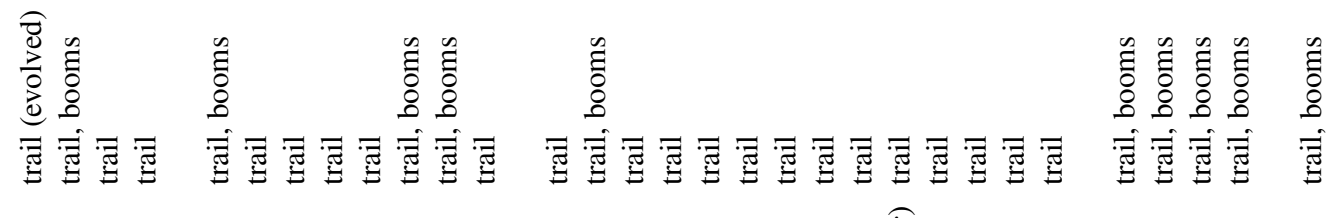

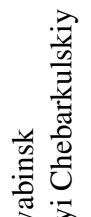

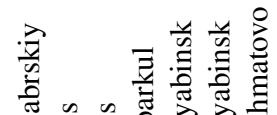

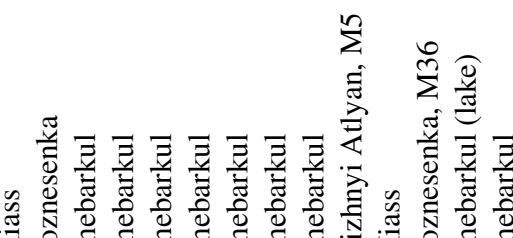

$\sum$

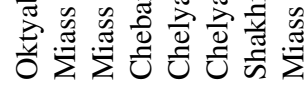

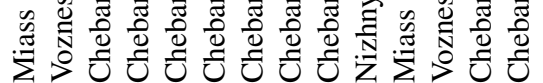

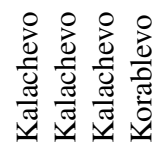

要

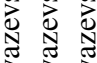

䆜

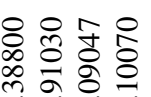

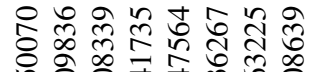

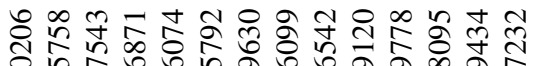

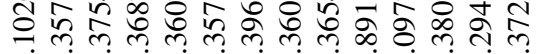

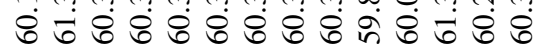

क्टेत्त̄

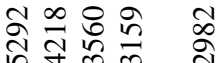

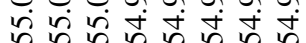

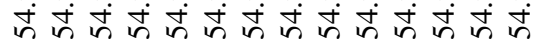

के के ऊू

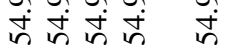
88 


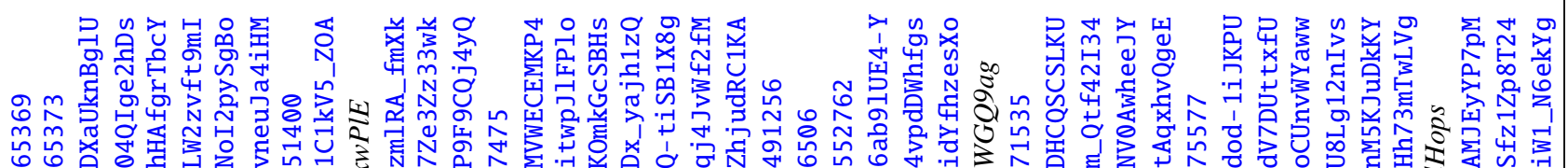

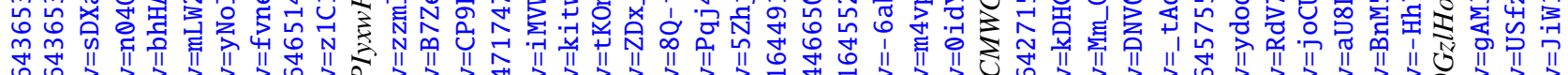

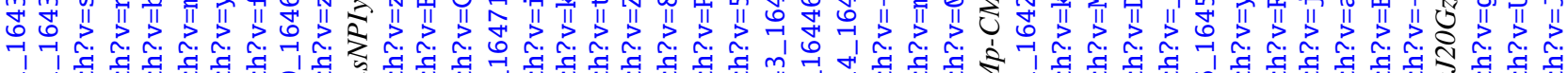
1

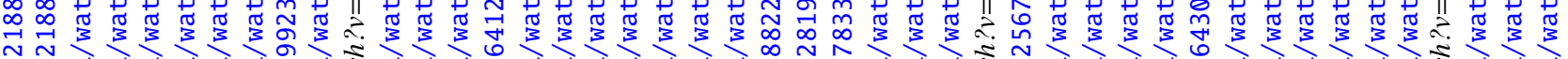

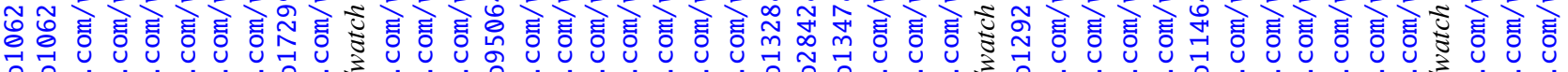

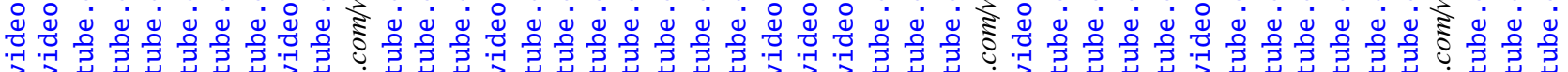
>之

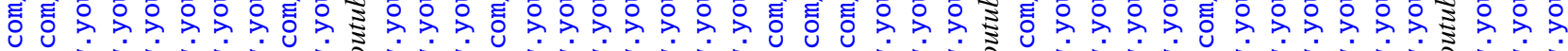

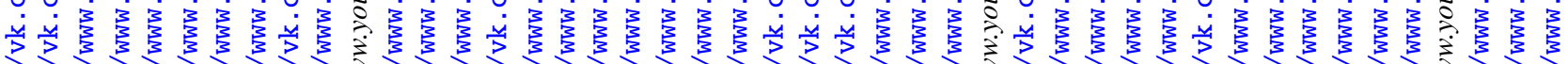

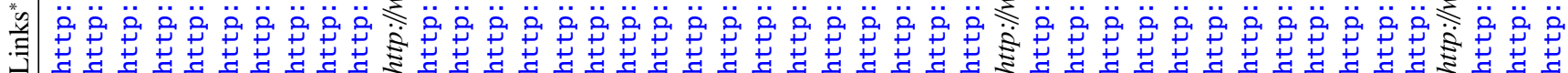

.ี

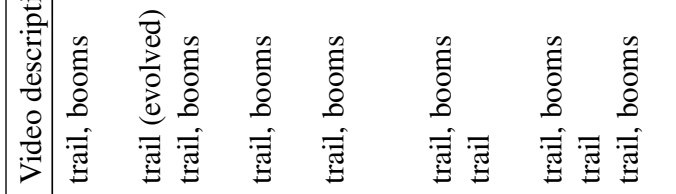

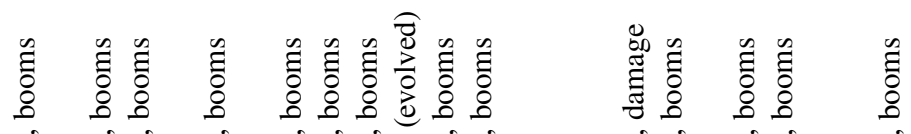

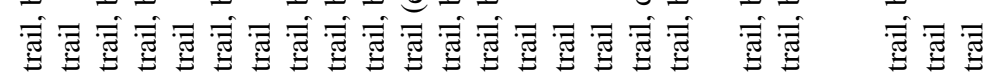

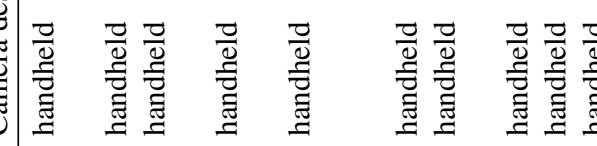

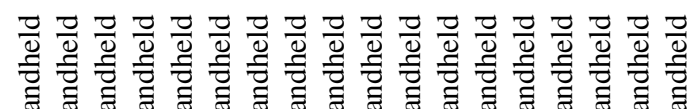
翌咅

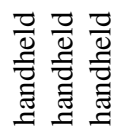
긍 离

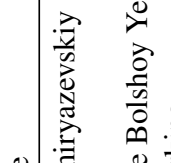

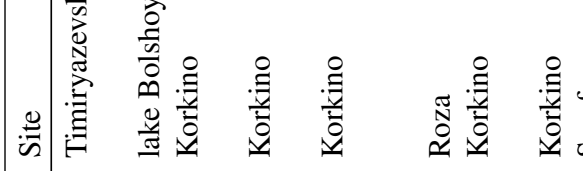

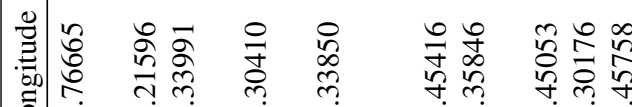
i。

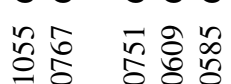
年字 :

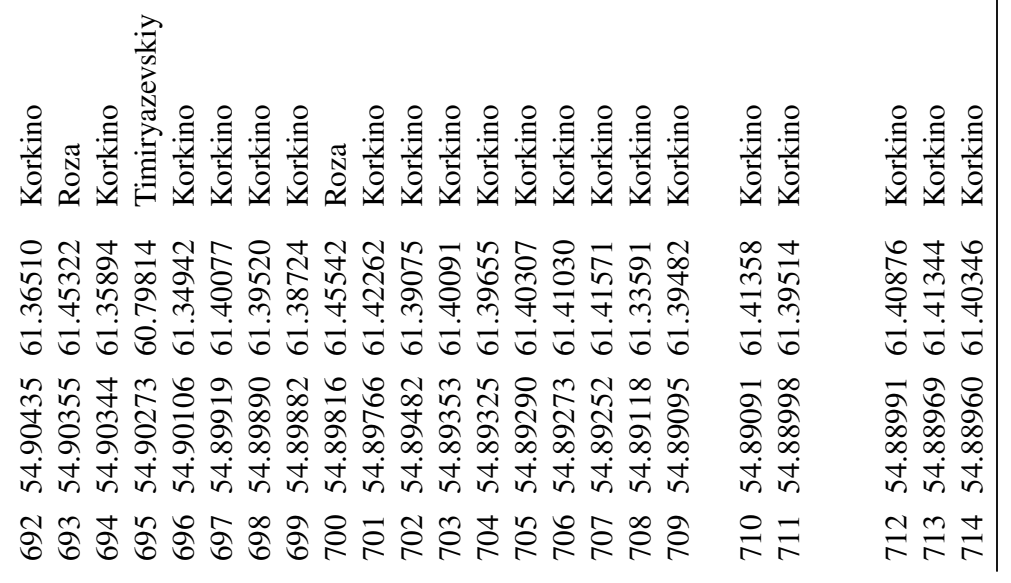


J. Borovička et al.: A catalog of video records of the 2013 Chelyabinsk superbolide

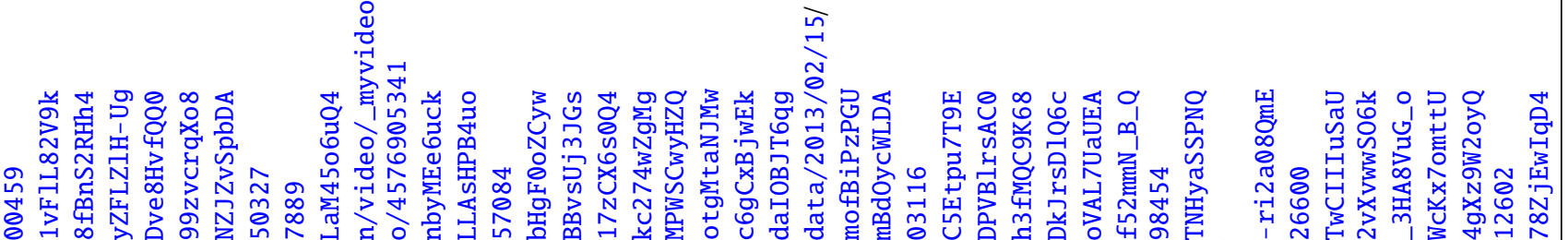

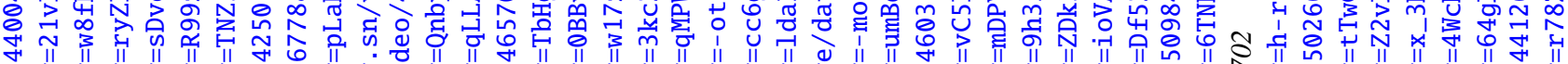

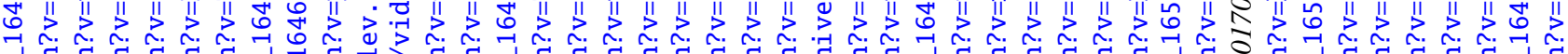
1

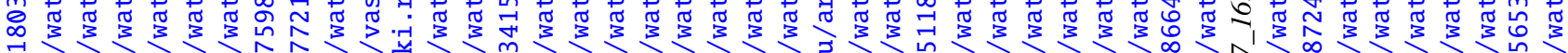

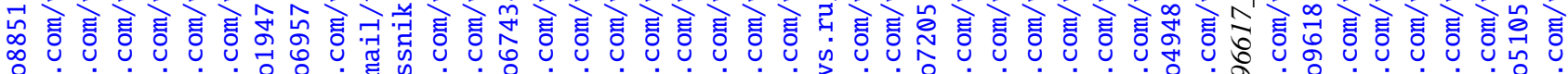
ष்

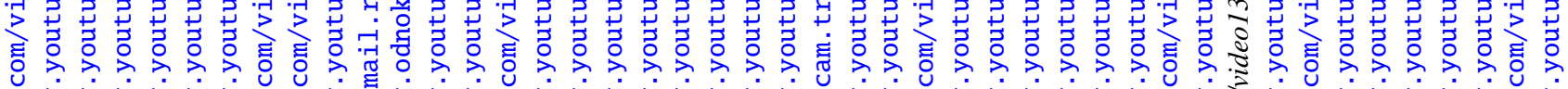

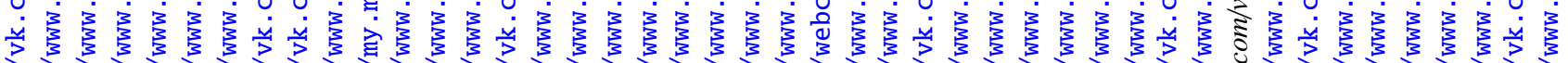

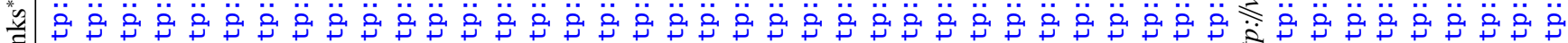

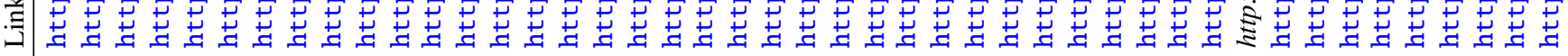

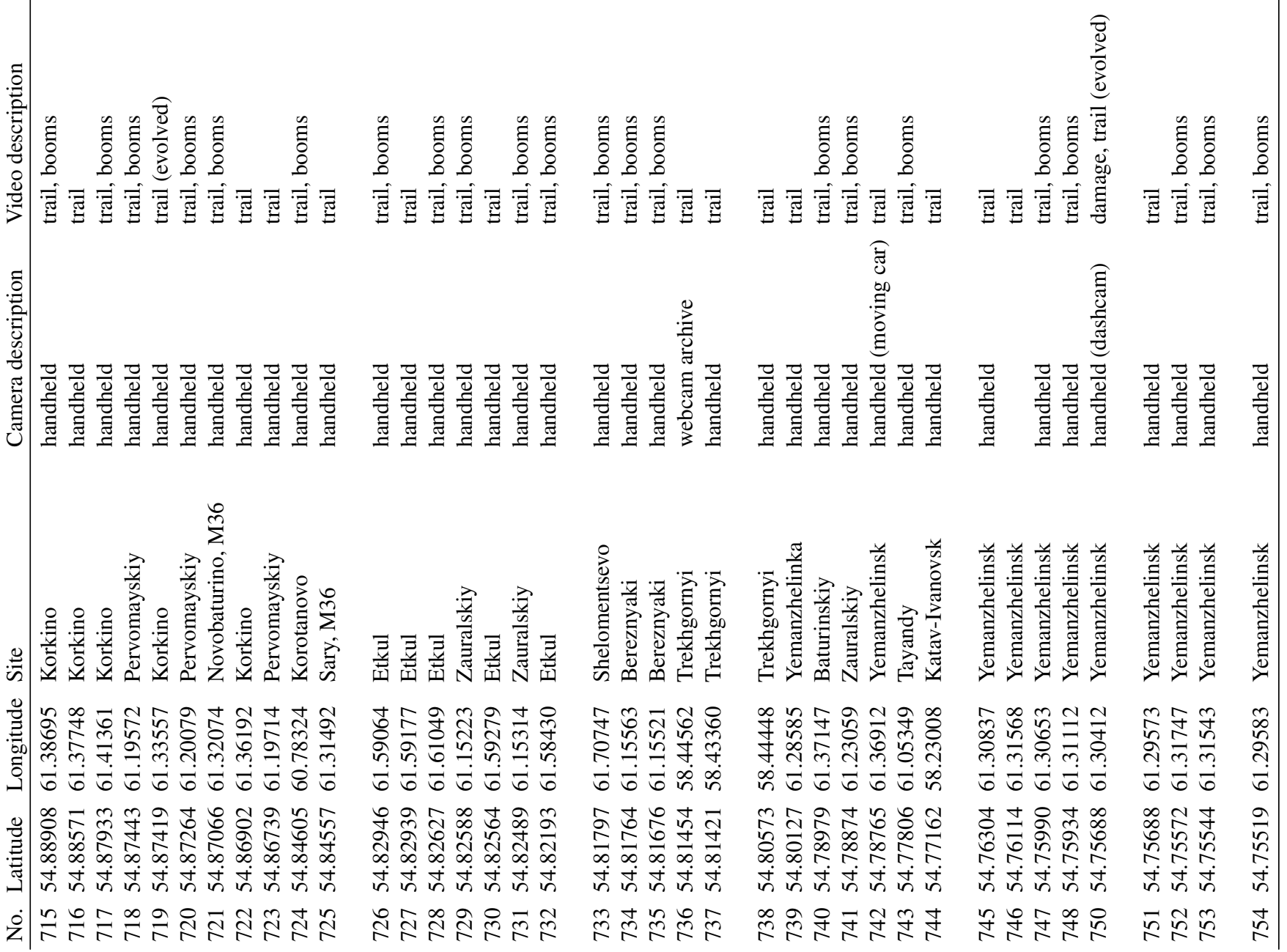




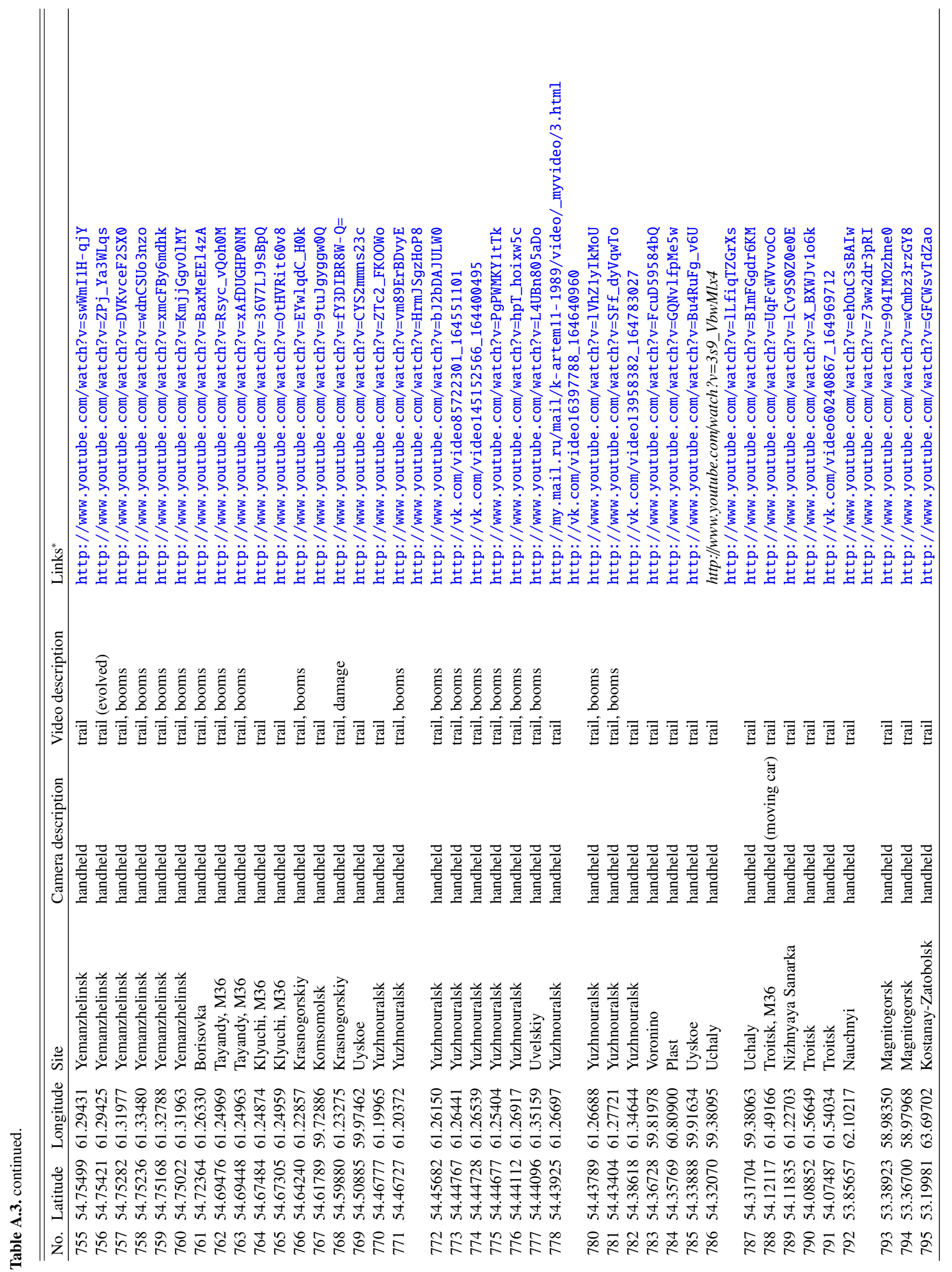


J. Borovička et al.: A catalog of video records of the 2013 Chelyabinsk superbolide

焉

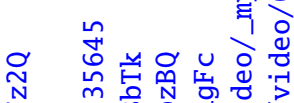

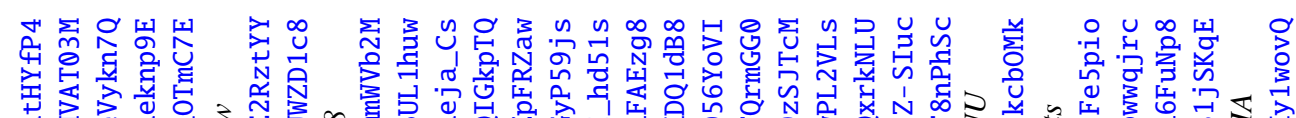

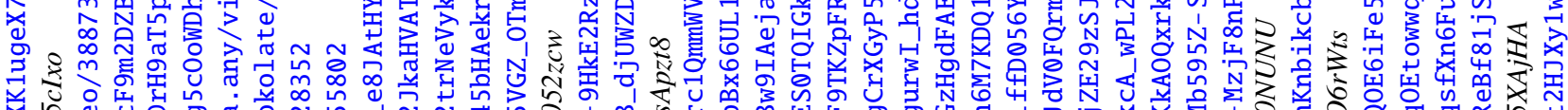

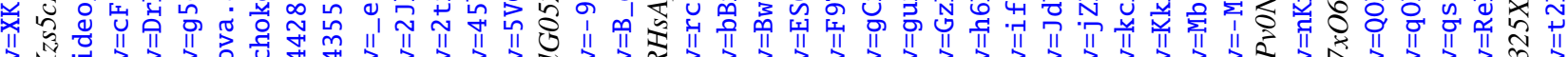

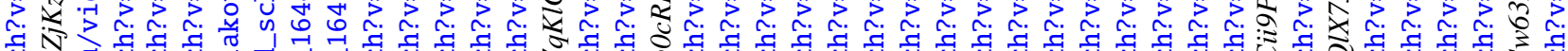

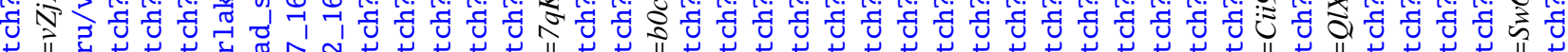

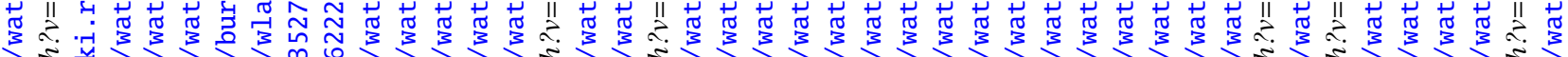

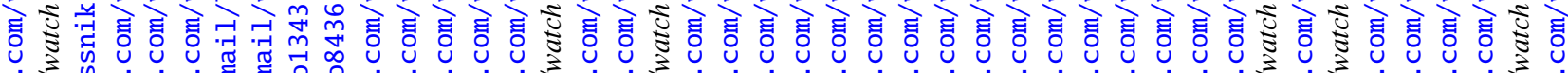
\&

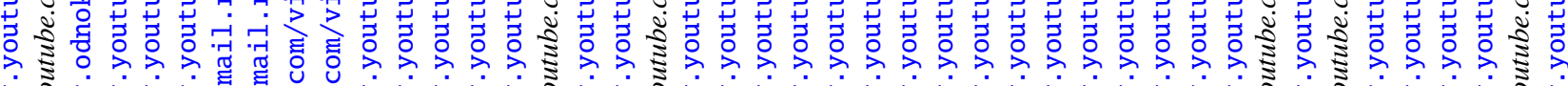

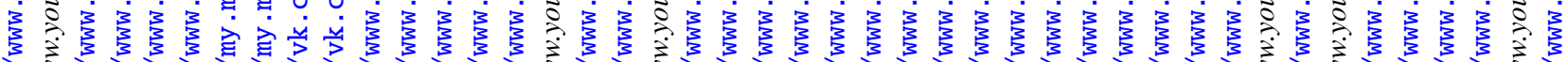
严

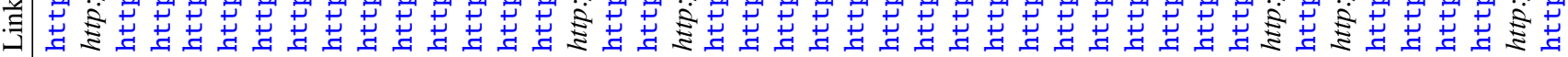

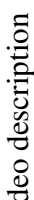

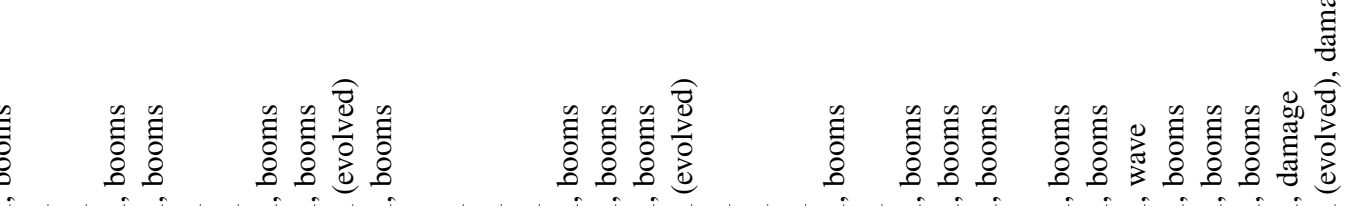

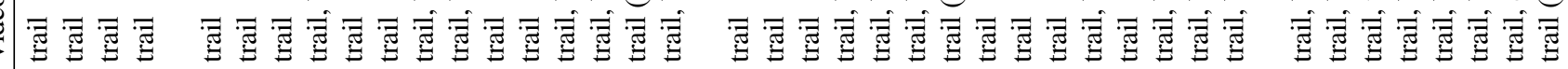

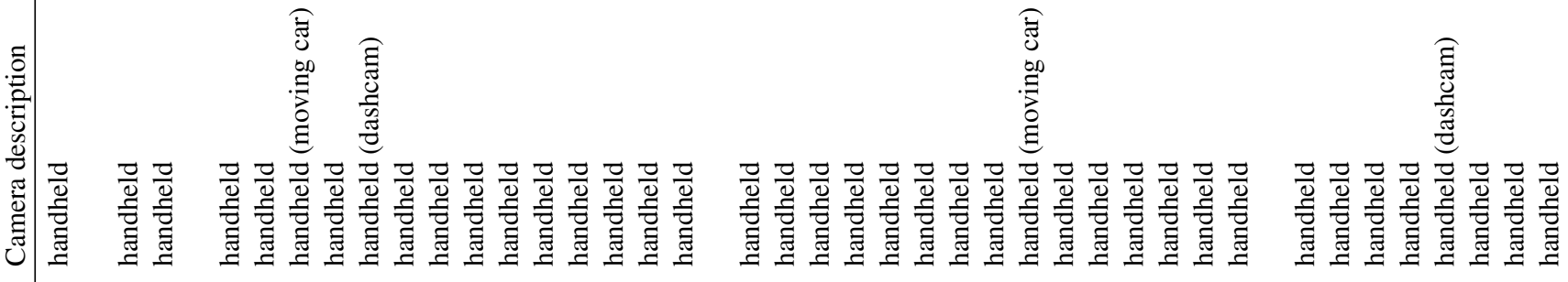




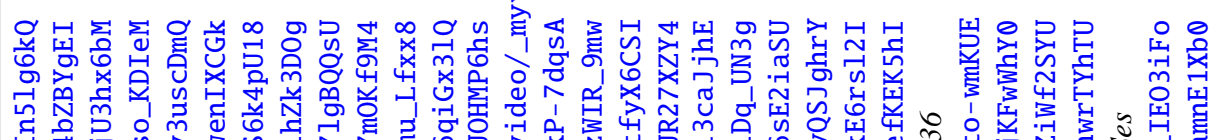

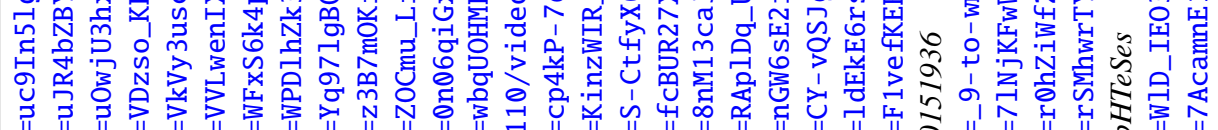

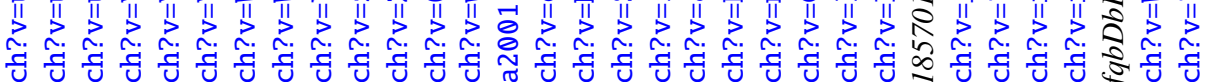

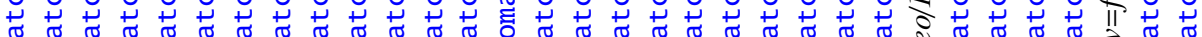

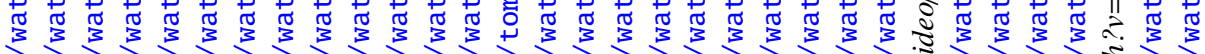

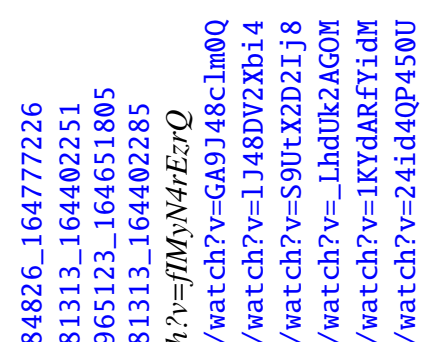

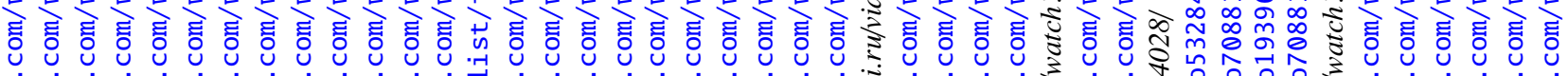

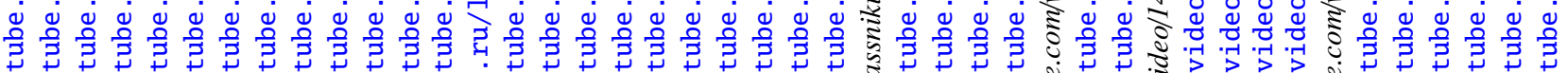

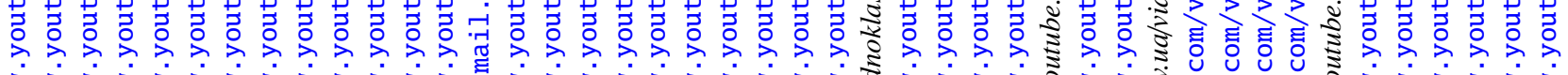

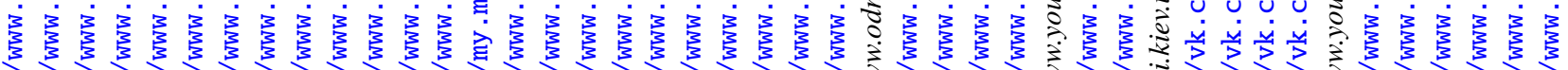

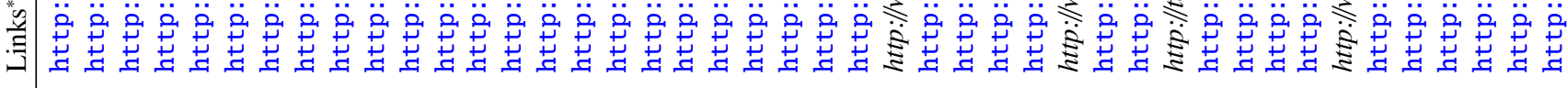

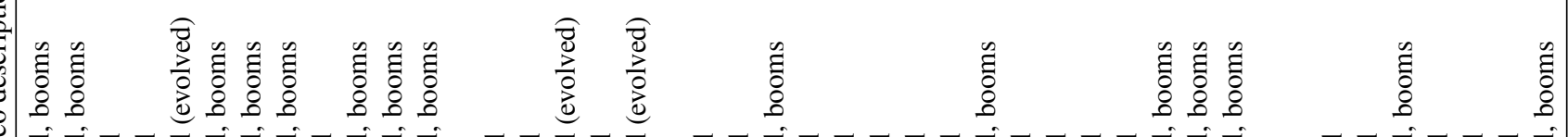

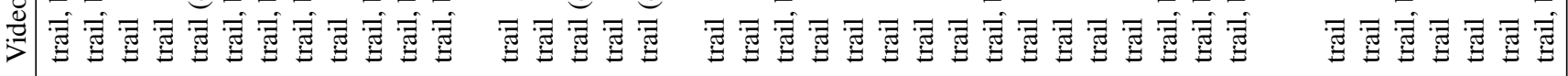

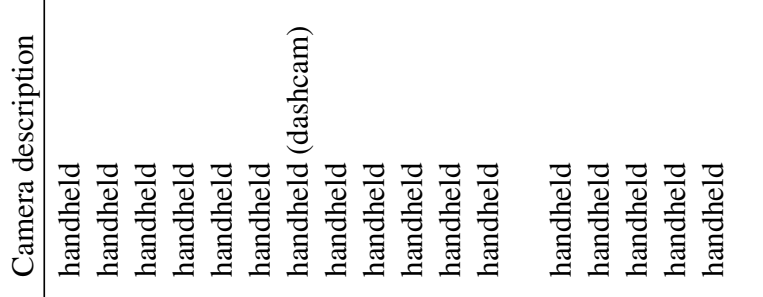

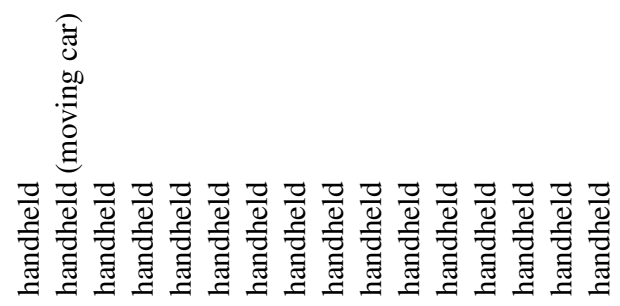

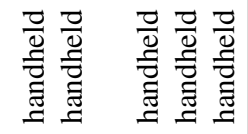

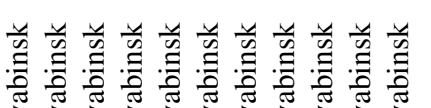

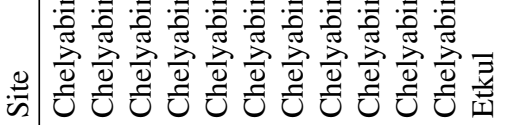

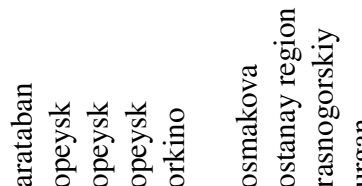

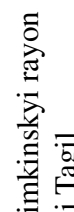<smiles>CCCCC</smiles>

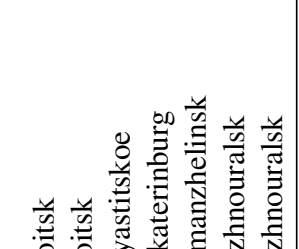

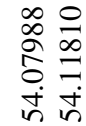

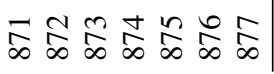


J. Borovička et al.: A catalog of video records of the 2013 Chelyabinsk superbolide

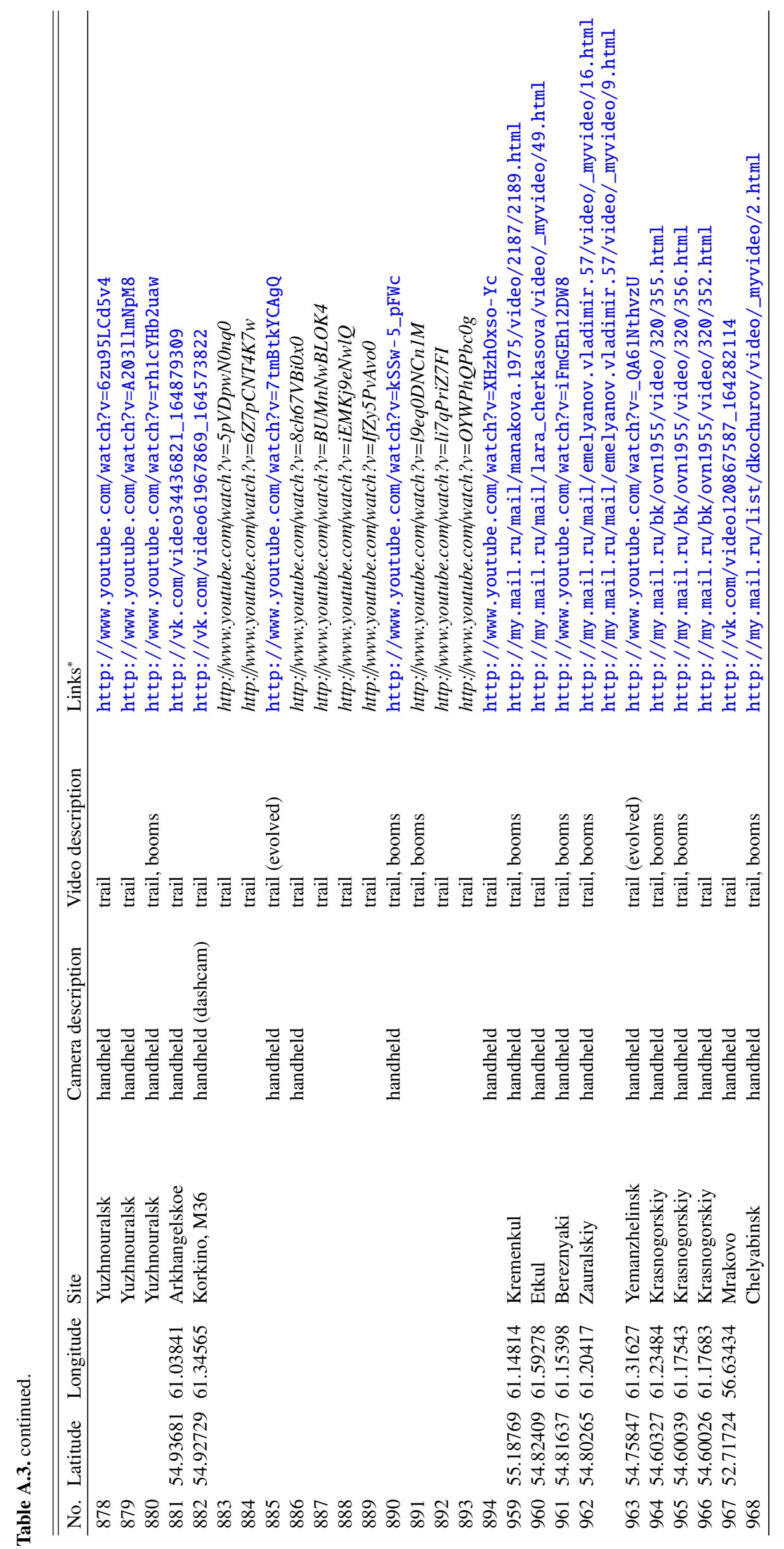




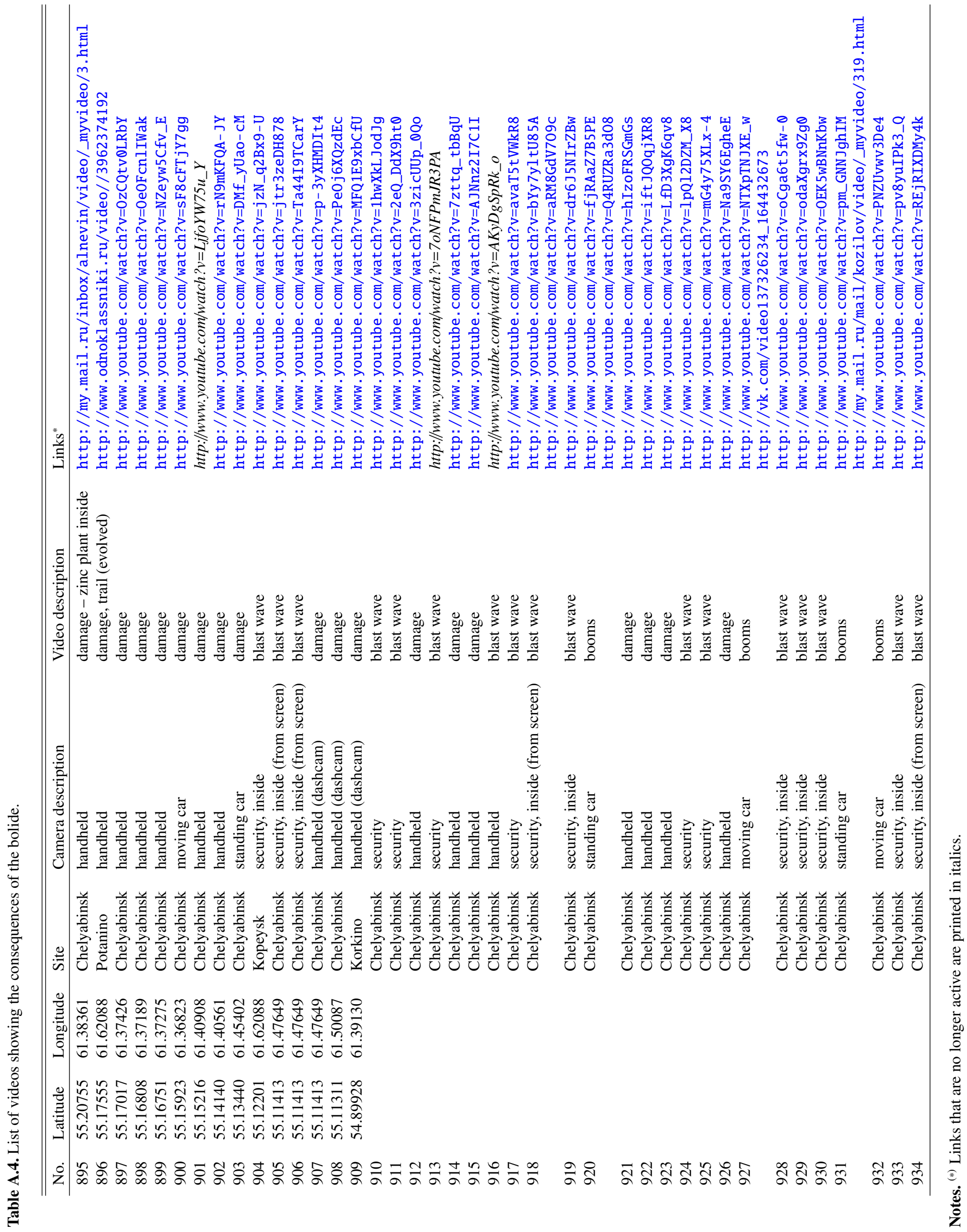




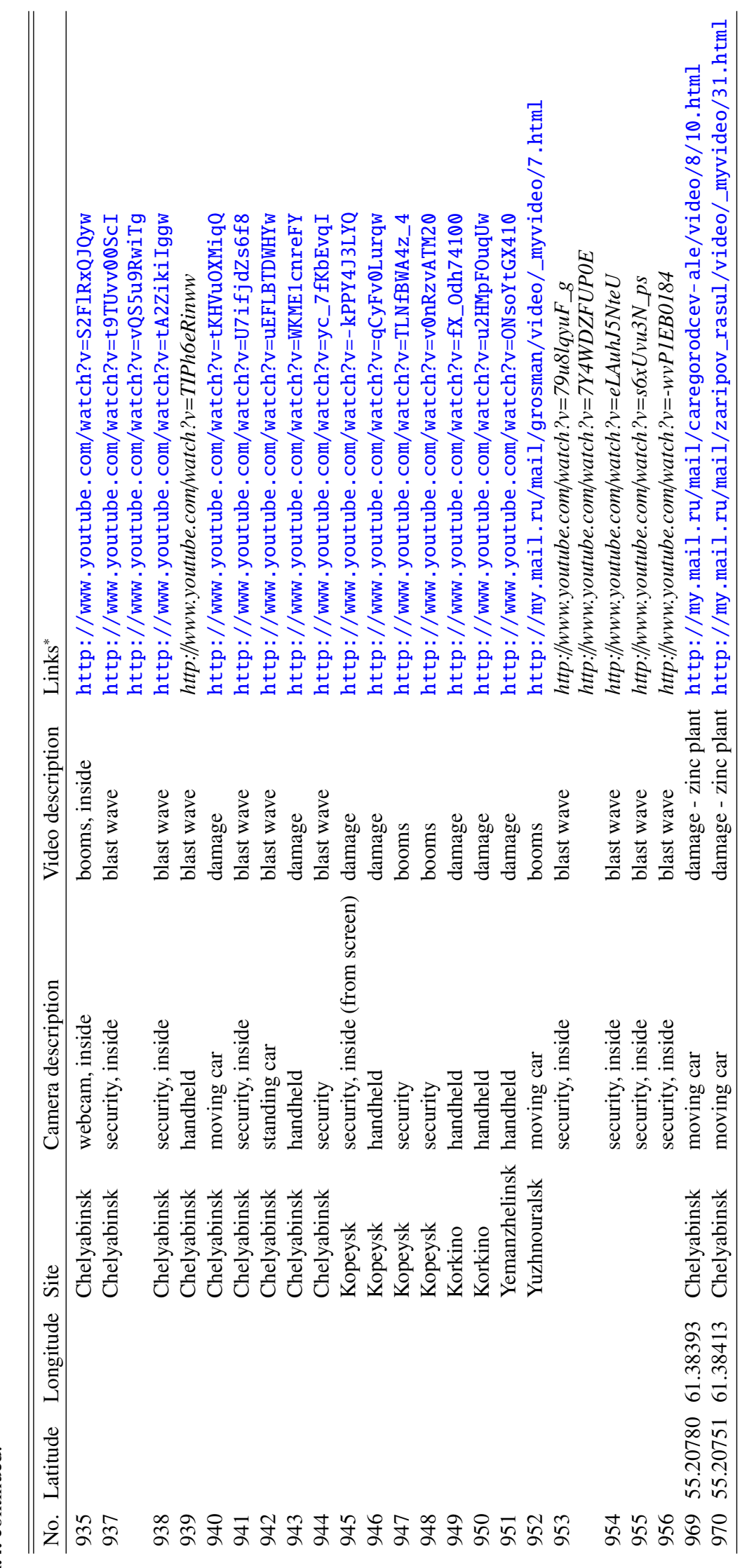

